\title{
Aeroelastic Simulation Using CFD/CSD Coupling Based on Precise Integration Method
}

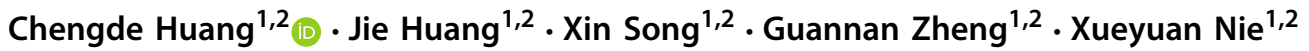

Received: 4 July 2019 / Revised: 6 January 2020 / Accepted: 7 January 2020

(c) The Korean Society for Aeronautical \& Space Sciences 2020

\begin{abstract}
Aeroelasticity studies the interaction between the aerodynamic loads and the flexible structures, and has gained much attention in the design of modern aircraft. Most of the existing computational fluid dynamics/computational structural dynamics (CFD/CSD) coupling approaches are based on Runge-Kutta method, central difference method, linear multi-step method and so on, which are conditionally stable and are unsuitable for the stiff problem that requires a very small time step to solve. In this paper, the precise integration method (PIM) formula is derived for the structural modal equations and then the PIM-based CFD/CSD coupling method is presented. The three-dimensional AGARD wing 445.6 and a sweptback wing are considered here for aeroelastic studies. The flutter results demonstrate that the presented method is comparable in accuracy to the traditional strong coupling method and has better numerical stability property than some exiting improved methods. For the static aeroelastic analyses, applying a large damping ratio to the structural equations helps to obtain the equilibrium quickly but may lead to the stiff problem, which was seldom discussed before. The results show that the presented PIM-based coupling method can overcome the stiff problem arising in static aeroelastic systems and is more efficient than the traditional coupling approach based on Runge-Kutta method, especially when a large damping ratio is applied.
\end{abstract}

Keywords Flutter $\cdot$ Static aeroelasticity $\cdot$ CFD/CSD coupling $\cdot$ Precise integration method

\section{List of Symbols}

$\begin{array}{ll}\eta_{i} & \text { Generalized displacement } \\ \xi_{i} & \text { Damping ratio } \\ \omega_{i} & \text { Natural frequency } \\ Q_{i} & \text { Generalized force } \\ y^{+} & \text {Wall } y \text {-plus } \\ j & \text { Imaginary unit } \\ i, n & \text { Index } \\ r & \text { Stiffness ratio } \\ \lambda_{1} \lambda_{2} & \text { Eigenvalue } \\ \eta & \text { Vector of generalized displacements } \\ \boldsymbol{C} & \text { Damping matrix } \\ \boldsymbol{\Lambda} & \text { Spectral matrix } \\ k & \text { The number of modes }\end{array}$

Guannan Zheng

zhengguannan@imech.ac.cn

1 Institute of Mechanics, Chinese Academy of Sciences, Beijing 100190, China

2 School of Engineering Science, University of Chinese Academy of Sciences, Beijing 100049, China

$\begin{array}{ll}\boldsymbol{x} & \text { State vector } \\ \boldsymbol{H} & \text { State matrix } \\ \boldsymbol{I} & \text { Unit matrix } \\ \boldsymbol{f}(t) \boldsymbol{Q}(t) & \text { Force vector } \\ t & \text { Time } \\ \boldsymbol{T} & \text { Exponential matrix } \\ m & N, M \text {, integer } \\ \Delta t & \text { Time step } \\ \varepsilon & \text { Small number } \\ \alpha & \text { Coefficient } \\ x, y, z & \text { Coordinate } \\ d & \text { Support radius } \\ \phi & \text { Radial basis function } \\ b & \text { Semi-chord } \\ \omega & \text { Natural frequency of the second mode } \\ \mu & \text { Mass ratio }\end{array}$

\section{Introduction}

Flutter is a dynamic instability of a fluid structure coupling system and is one of the major concerns in the design of 
modern aircraft. Doublet lattice method (DLM) [1] is a frequency domain method and has been widely used in the flutter calculations due to its high efficiency [2-9]. However, DLM is based on the linearized potential theory in the subsonic condition, so it cannot simulate transonic or viscous flows. Computational fluid dynamics/computational structural dynamics (CFD/CSD) coupling approach predicts flutter boundaries in time domain and can account for the aerodynamic nonlinearities. The CFD/CSD coupling strategies can be classified into the monolithic approach and the partitioned approach [10]. The monolithic approach uses the integrated aero-structure solver whose governing equations are highly nonlinear and difficult to solve, so it is seldom applied to engineering problems, while the partitioned approach allows the usage of exiting solvers and is widely adopted in practice [11].

The partitioned approach needs additional data communications at the fluid-structure interface and can be further divided into the weak coupling and the strong coupling. For a weakly coupled code, the aerodynamic forces and structural displacements are exchanged between the CFD and CSD solvers only once, so the existence of time synchronization problem is inevitable and the coupling scheme is only first-order accurate in time [12-15]. To improve the time accuracy, some improved methods have been studied. For example, Lesoinne, Farhat [16] studied the improved weak coupling schemes based on mid-step to alleviate the time synchronization problem and improve the accuracy. Zhang et al. [17] proposed two better weak coupling algorithms having second-order time accuracy based on the polynomial extrapolation of generalized aerodynamic forces. Hou et al. [18] reviewed some algorithms that could achieve high accuracy by solving the fluid equations only once within each time step.

The strong coupling strategy performs data communications several times within each physical time step and usually has two models. One of the models uses the dualtime formulation that a pseudo-time-derivative term is added to the $\mathrm{N}-\mathrm{S}$ equations and structural equations simultaneously, and the data communications are performed on pseudo-time level as shown in Fig. 1 [19-21]. After some subiterations, the pseudo-time-derivative term vanishes and the coupling scheme can be second-order accurate in time. However, this scheme needs CFD mesh motion at each subiteration and therefore is computationally expensive [13]. Figure 2 shows the other strong coupling model which is implemented on physical time level $[22,23]$. This model allows the fluid equations and structural equations to use different time marching schemes and can be implemented between various CFD and CSD solvers without much work.

For aeroelastic systems, the structural equations of motion are usually solved by Runge-Kutta method [14, 15, 24], central difference method [12, 16], linear multi-step method [17]

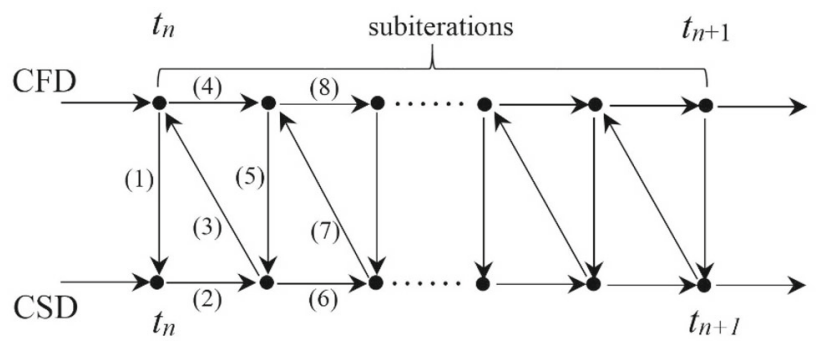

Fig. 1 Strong coupling on pseudo-time level

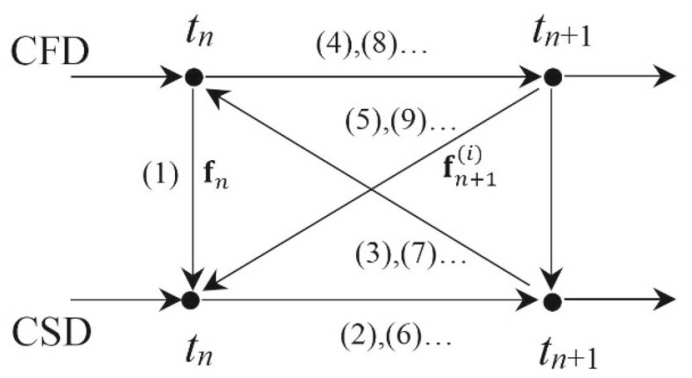

Fig. 2 Strong coupling on physical time level

and so on. However, all these methods may lead to errors and numerical difficulties [25-27] such as the stiff problem, for which some numerical methods are numerically unstable unless the time step size is set to a very small value. Instead of using finite difference methods, Zhong [26] proposed the precise integration method (PIM) to solve ordinary differential equations. For linear time-invariant systems, the numerical results of PIM can approach computer precision. Moreover, PIM is explicit, unconditionally stable and free from the stiff problem. Therefore, PIM is gradually used in engineering problems such as structural dynamics, soil mechanics, random vibrations, multibody systems and so on [28].

A system of ordinary differential equations is stiff if the ratio between the largest and the smallest eigenvalue is large. The stiff equations are characterized by having rapidly changing transients and slowly changing transients simultaneously. For some numerical approaches, the rapidly changing transients require the time step size to be extremely small, otherwise the numerical instability occurs [27, 29]. Even if the solution curve at the steady state is flat and smooth, the time step size is still limited to an unreasonably small value, which leads to low computational efficiency.

The flexibility method is well known for the static aeroelastic calculations [30]. However, it is found from engineering applications that if the quality of the structural FEM model is bad or the interpolation nodes on the structural surface is not chosen suitably, the flexibility method may induce local distortion. This is because the flexibility matrix contains all the natural modes of a discretized structure and the local distortion is associated with the high-order modes, which may be triggered by the concentrated forces. This difficulty 
can be overcome using the modal approach, which only considers some low-order modes and excludes the high-order modes. When the modal approach is used for static aeroelastic simulations, the damping ratio is suggested to be applied to the structural equations of motion so that the steady state can be obtained quickly and then the computational cost is reduced. Usually, a large damping ratio helps to obtain the static aeroelastic equilibrium quickly. However, the stiffness ratio increases as the damping ratio increases. Therefore, if the damping ratio is taken to be too large, the structural equations of motion will become a system of stiff equations so that the computational efficiency becomes very low. Since the stiff problem was seldom discussed in aeroelastic applications, it is necessary to develop an effective and efficient coupling approach to overcome the stiff problem.

Most of the existing CFD/CSD coupling approaches are based on conventional numerical methods [14-17] that are conditionally stable and are unsuitable for the stiff problem. To solve this problem, the PIM formula is derived and then the PIM-based CFD/CSD coupling approach is presented. Both flutter and static aeroelasticity are analyzed in this paper. The time accuracy, efficiency and numerical stability of the presented method will be discussed.

\section{Numerical Approach}

\subsection{Aerodynamic Solver}

The aerodynamic forces are calculated by ANSYS FLUENT 14.5 software which is a finite volume Navier-Stokes solver. In this paper, the gradients are evaluated by the GreenGauss scheme, and the turbulence is simulated using the one equation Spalart-Allmaras model. The second-order upwind scheme is used for the spatial discretization of the flow variables and the turbulent viscosity.

The time-dependent Navier-Stokes equations are integrated in time using the dual-time formulation suggested by Jameson [31]. A pseudo-time-derivative term is introduced into the equations and is discretized using the first-order Euler scheme. The time-dependent term is discretized implicitly by a three-point backward difference scheme. The discretized equations are solved by the Gauss-Seidel algorithm. The pseudo-time-derivative term vanishes at the end of each physical time step after a series of subiterations and then the aerodynamic calculation is second-order time accuracy [ 19 , 20, 32].

For aeroelastic simulations, the solution of structural displacements and the mesh deformation method are implemented into the CFD solver through the user-defined functions, which are written in the $\mathrm{C}$ programming language and can be loaded with the software to extend its range of application.

\subsection{Structural Equations}

The modal equation has the following form for the $i$-th mode:

$\frac{d^{2} \eta_{i}}{d t^{2}}+2 \xi_{i} \omega_{i} \frac{d \eta_{i}}{d t}+\omega_{i}^{2} \eta_{i}=Q_{i}$

where $\eta_{i}$ is the generalized displacement, $\xi_{i}$ is the damping ratio, $\omega_{i}$ is the natural frequency, and $Q_{i}$ is the generalized force. If $0<\xi_{i} \leq 1$, then the eigenvalues are:

$\lambda_{1}=-\xi_{i} \omega_{i}+\omega_{i} \sqrt{1-\xi_{i}^{2}} j$

$\lambda_{2}=-\xi_{i} \omega_{i}-\omega_{i} \sqrt{1-\xi_{i}^{2}} j$,

where $j^{2}=-1$. The stiffness ratio is defined as:

$r=\frac{\operatorname{Re}\left(-\lambda_{2}\right)}{\operatorname{Re}\left(-\lambda_{1}\right)}=\frac{\xi_{i} \omega_{i}}{\xi_{i} \omega_{i}}=1$.

If $\xi_{i}>1$, then the eigenvalues are:

$\lambda_{1}=-\xi_{i} \omega_{i}+\omega_{i} \sqrt{\xi_{i}^{2}-1}$,
$\lambda_{2}=-\xi_{i} \omega_{i}-\omega_{i} \sqrt{\xi_{i}^{2}-1}$.

The stiffness ratio is:

$r=\frac{\operatorname{Re}\left(-\lambda_{2}\right)}{\operatorname{Re}\left(-\lambda_{1}\right)}=\frac{\xi_{i}+\sqrt{\xi_{i}^{2}-1}}{\xi_{i}-\sqrt{\xi_{i}^{2}-1}}=-1+\frac{2}{1-\sqrt{1-\frac{1}{\xi_{i}^{2}}}}$.

Therefore, the stiffness ratio $r$ increases with $\xi_{i}$ on the interval $(1,+\infty)$. Figure 3 plots the curve of stiffness ratio versus damping ratio, showing that $r$ increases with $\xi_{i}$ rapidly when $\xi_{i}$ is greater than 1 . Especially, the stiffness ratio $r$ is 10 if $\xi_{i}$ is set to 1.739 , while the stiffness ratio $r$ will increase to 100 if $\xi_{i}$ increases to 5.05. Usually, if $r \geq 10$, the differential equation can be considered as a stiff equation. If some conventional numerical methods (e.g., Runge-Kutta method and linear multi-step method) are used to solve the stiff problem, the time step satisfying the numerical stability condition will be much less than the non-stiff problem so that the computational cost is expensive. Since all these methods are unsuitable for the stiff problem, PIM which is an explicit but unconditionally stable method is employed to solve it in this paper.

Equation (1) can be expressed as the matrix form:

$\frac{d^{2} \eta}{d t^{2}}+C \frac{d \eta}{d t}+\Lambda \eta=Q$

where $\boldsymbol{C}=\operatorname{diag}\left\{2 \xi_{1} \omega_{1}, \ldots, 2 \xi_{k} \omega_{k}\right\}$ denotes the damping matrix, $\boldsymbol{\Lambda}=\operatorname{diag}\left\{\omega_{1}^{2}, \ldots, \omega_{k}^{2}\right\}$ denotes the spectral matrix, 


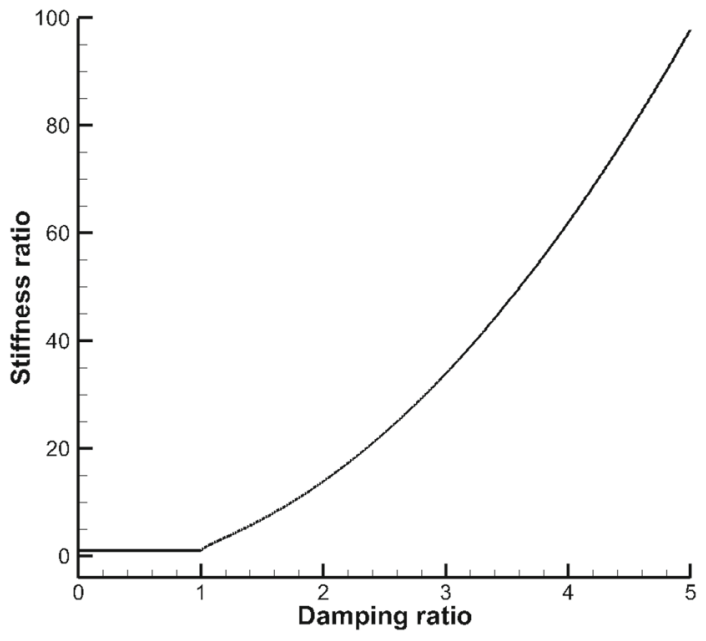

Fig. 3 Stiffness ratio versus damping ratio

$k$ denotes the number of modes extracted. Equation (6) can be further rewritten as the state form:

$\frac{d x}{d t}=\boldsymbol{H} \boldsymbol{x}+\boldsymbol{f}(t)$,

where

$\boldsymbol{x}=\left[\begin{array}{r}\eta \\ \frac{d \boldsymbol{\eta}}{d t}\end{array}\right], \quad \boldsymbol{f}(t)=\left[\begin{array}{r}\mathbf{0} \\ \boldsymbol{Q}(t)\end{array}\right], \quad \boldsymbol{H}=\left[\begin{array}{cc}\mathbf{0} & \boldsymbol{I}_{k \times k} \\ -\boldsymbol{\Lambda} & -\boldsymbol{C}\end{array}\right]$.

\subsection{CFD/CSD Coupling Using PIM}

Given that the coefficient matrices of the modal equations [Eq. (6)] are diagonal, the recursion formula [Eq. (13)] derived in this section has less computational effort than the original PIM formula by Zhong [26]. According to the theory of ordinary differential equations, the analytical solution of Eq. (7) can be expressed as:

$\boldsymbol{x}(t)=e^{\boldsymbol{H}\left(t-t_{0}\right)} \boldsymbol{x}_{0}+\int_{t_{0}}^{t} e^{\boldsymbol{H}(t-\xi)} \boldsymbol{f}(\xi) \mathrm{d} \xi$.

Let $t=t_{n+1}$,

$$
\begin{aligned}
\boldsymbol{x}\left(t_{n+1}\right)= & e^{\boldsymbol{H}\left(t_{n}+\Delta t-t_{0}\right)} \boldsymbol{x}_{0}+\int_{t_{0}}^{t_{n}} e^{\boldsymbol{H}\left(t_{n}+\Delta t-\xi\right)} \boldsymbol{f}(\xi) \mathrm{d} \xi \\
& +\int_{t_{n}}^{t_{n+1}} e^{\boldsymbol{H}\left(t_{n+1}-\xi\right)} \boldsymbol{f}(\xi) \mathrm{d} \xi \\
= & \boldsymbol{T} \boldsymbol{x}\left(t_{n}\right)+\int_{t_{n}}^{t_{n+1}} e^{\boldsymbol{H}\left(t_{n+1}-\xi\right)} \boldsymbol{f}(\xi) \mathrm{d} \xi
\end{aligned}
$$

where $\boldsymbol{T}$ is the exponential matrix:

$$
\boldsymbol{T}=e^{\boldsymbol{H} \Delta t}=\sum_{i=0}^{\infty} \frac{(\boldsymbol{H} \Delta t)^{i}}{i !} .
$$

In an aeroelastic calculation, $\boldsymbol{f}(t)$ denotes aerodynamic forces and its analytical expression is not available on the interval $\left(t_{n}, t_{n+1}\right)$. To compute the integration in Eq. (10), an approximation should be given. In the present work, the linear interpolation is used:

$f(t) \approx f_{n}+\frac{f_{n+1}-f_{n}}{\Delta t}\left(t-t_{n}\right)$.

By substituting Eq. (12) into Eq. (10) and calculating the integration, the following formulation is obtained:

$$
\begin{aligned}
& \boldsymbol{x}_{n+1} \\
& =\boldsymbol{T}\left(\boldsymbol{x}_{n}-\left[\begin{array}{c}
\boldsymbol{\Lambda}^{-1} \boldsymbol{Q}_{n}-\boldsymbol{\Lambda}^{-1} \boldsymbol{C} \boldsymbol{\Lambda}^{-1}\left(\boldsymbol{Q}_{n+1}-\boldsymbol{Q}_{n}\right) / \Delta t \\
\boldsymbol{\Lambda}^{-1}\left(\boldsymbol{Q}_{n+1}-\boldsymbol{Q}_{n}\right) / \Delta t
\end{array}\right]\right) \\
& +\left[\begin{array}{c}
\boldsymbol{\Lambda}^{-1} \boldsymbol{Q}_{n+1}-\boldsymbol{\Lambda}^{-1} \boldsymbol{C} \boldsymbol{\Lambda}^{-1}\left(\boldsymbol{Q}_{n+1}-\boldsymbol{Q}_{n}\right) / \Delta t \\
\boldsymbol{\Lambda}^{-1}\left(\boldsymbol{Q}_{n+1}-\boldsymbol{Q}_{n}\right) / \Delta t
\end{array}\right] \\
& =\boldsymbol{\Phi}\left(\boldsymbol{x}_{n}, \boldsymbol{Q}_{n}, \boldsymbol{Q}_{n+1}\right) .
\end{aligned}
$$

The linear interpolation can ensure this formulation has second-order time accuracy and is sufficient for accuracy in present work. It is noted that the aerodynamic forces at $t_{n+1}$ are unknown at the beginning of each time interval. To compute $\boldsymbol{x}_{n+1}$ and $\boldsymbol{Q}_{n+1}$, the strong coupling model (Fig. 2) is used which contains an iteration process on the physical time level. Let

$\boldsymbol{x}_{n+1}^{(i)}=\boldsymbol{\Phi}\left(\boldsymbol{x}_{n}, \boldsymbol{Q}_{n}, \boldsymbol{Q}_{n+1}^{(i)}\right)$,

where $i$ denotes the iteration index. At the beginning of each time interval, let $i=1$ and $\boldsymbol{Q}_{n+1}^{(i)}=\boldsymbol{Q}_{n}$, then compute $\boldsymbol{x}_{n+1}^{(i)}$ according to Eq. (14). The structural displacements are then transferred to the CFD solver to update the CFD mesh and compute the new generalized aerodynamic forces $\boldsymbol{Q}_{n+1}^{(i+1)}$, which is then substituted into Eq. (14) to recalculate $\boldsymbol{x}_{n+1}^{(i+1)}$. Usually three iterations are enough to obtain a converged result.

Additionally, the exponential matrix $\boldsymbol{T}$ needs to be evaluated. PIM suggests a highly accurate approach to compute $\boldsymbol{T}$. Let

$\boldsymbol{T}=e^{\boldsymbol{H} \Delta t}=\left(e^{\boldsymbol{H} \Delta t / m}\right)^{m}=\left(e^{\boldsymbol{H} \varepsilon}\right)^{m}$,

where $m$ is a large integer. It is suggested to select $m=2^{N}$ and $N=20$ such that $\varepsilon=\Delta t / m$ is an extremely small number. The 
first five terms of Taylor expansion are used to approximate $e^{\boldsymbol{H} \varepsilon}$ :

$$
\begin{aligned}
e^{\boldsymbol{H} \varepsilon} & =\boldsymbol{I}+\boldsymbol{H} \varepsilon+\frac{1}{2}(\boldsymbol{H} \varepsilon)^{2}+\frac{1}{3 !}(\boldsymbol{H} \varepsilon)^{3}+\frac{1}{4 !}(\boldsymbol{H} \varepsilon)^{4} \\
& =\boldsymbol{I}+\boldsymbol{T}_{\mathrm{a}}
\end{aligned}
$$

Then,

$$
\begin{aligned}
\boldsymbol{T} & =\left(\boldsymbol{I}+\boldsymbol{T}_{\mathrm{a}}\right)^{2^{N}}=\left(\boldsymbol{I}+\boldsymbol{T}_{\mathrm{a}}\right)^{2^{N-1}}\left(\boldsymbol{I}+\boldsymbol{T}_{\mathrm{a}}\right)^{2^{N-1}} \\
& =\left[\left(\boldsymbol{I}+\boldsymbol{T}_{\mathrm{a}}\right)^{2^{N-2}}\left(\boldsymbol{I}+\boldsymbol{T}_{\mathrm{a}}\right)^{2^{N-2}}\right]\left[\left(\boldsymbol{I}+\boldsymbol{T}_{\mathrm{a}}\right)^{2^{N-2}}\left(\boldsymbol{I}+\boldsymbol{T}_{\mathrm{a}}\right)^{2^{N-2}}\right] \\
& =\ldots
\end{aligned}
$$

It is noted that $\left(\boldsymbol{I}+\boldsymbol{T}_{\mathrm{a}}\right)\left(\boldsymbol{I}+\boldsymbol{T}_{\mathrm{a}}\right)=\boldsymbol{I}+\left(2 \boldsymbol{T}_{\mathrm{a}}+\boldsymbol{T}_{\mathrm{a}} \boldsymbol{T}_{\mathrm{a}}\right)$, where the parenthesis on the right-hand side can be treated as a new matrix $\boldsymbol{T}_{\mathrm{a}}$. Therefore, the factorization in Eq. (17) should be repeated $N$ times and is equivalent to the following loop statement:

for $($ iter $=0 ;$ iter $<N ;$ iter ++$) \quad \boldsymbol{T}_{\mathrm{a}}=2 \boldsymbol{T}_{\mathrm{a}}+\boldsymbol{T}_{\mathrm{a}} \boldsymbol{T}_{\mathrm{a}}$.

After the execution of this statement, the exponential matrix can be obtained by $\boldsymbol{T}=\boldsymbol{I}+\boldsymbol{T}_{\mathrm{a}}$. For more details about the precise computation of exponential matrix please refer to Zhong [26] and Lin et al. [25].

\subsection{Mesh Deformation and Coupling Procedure}

The deformation of fluid-structure interface, due to the deflection of the structure, usually requires the update of the volume mesh. It is tedious to regenerate the entire mesh manually especially in the case of flutter simulations which require mesh update to be performed repeatedly. Mesh deformation is the preferred choice to update the CFD mesh automatically. In this paper, the radial basis function (RBF) method [11] is used to deform the mesh. This method is based on the spatial positions of nodes only and can be performed on arbitrary point clouds with no connectivity information required. Moreover, only simple matrix operations are required for the RBF method. The general form of $\mathrm{RBF}$ interpolation is:

$s(\boldsymbol{x})=\sum_{i=1}^{M} \alpha_{i} \phi\left(\left\|\boldsymbol{x}-\boldsymbol{x}_{i}\right\|\right)$,

where $\boldsymbol{x}=(x, y, z), \boldsymbol{x}-\boldsymbol{x}_{i}^{2}=\left(x-x_{i}\right)^{2}+\left(y-y_{i}\right)^{2}+\left(z-z_{i}\right)^{2}$, and $M$ is the number of control points on the surface. After the coefficients in Eq. (19) are calculated based on the known values at the control points, the substitution of a new spatial point $\boldsymbol{x}$ into Eq. (19) yields the interpolated value. The
Wendland's C2 function [11], one of the most used radial basis functions, is chosen here:

$$
\begin{aligned}
& \phi\left(\left\|\boldsymbol{x}-\boldsymbol{x}_{i}\right\|\right) \\
& =\left\{\begin{array}{ll}
\left(1-\left\|\boldsymbol{x}-\boldsymbol{x}_{i}\right\| / d\right)^{4}\left(4\left\|\boldsymbol{x}-\boldsymbol{x}_{i}\right\| / d+1\right), \quad\left\|\boldsymbol{x}-\boldsymbol{x}_{i}\right\| \leq d \\
0, & \left\|\boldsymbol{x}-\boldsymbol{x}_{i}\right\|>d
\end{array},\right.
\end{aligned}
$$

where $d$ represents the support radius of the radial influence range and is chosen to be a suitable value to consider enough points near the interface and exclude the points far away. The accuracy of Wendland's C2 function in interpolations has been evaluated by the previous study [30].

However, selecting all the surface points as control points will make calculations expensive. To reduce the computational cost, Rendall, Allen [33] proposed the 'greedy' algorithm to reduce the size of the control points, which sacrifices the accuracy of the deformation on the surface with an acceptable error. In this work, the RBF method is utilized along with the 'greedy' algorithm to deform the CFD volume mesh.

The related techniques have been discussed. The procedure of the $\mathrm{CFD} / \mathrm{CSD}$ coupling simulation is summarized as follows:

1. Construct the structural finite element method (FEM) model and generate the CFD mesh.

2. Compute the modal data based on the structural FEM model.

3. Compute the unsteady aerodynamic loads using CFD methods.

4. Interpolate the forces from aerodynamic nodes onto structural nodes.

5. Solve the modal equations using PIM and obtain the structural displacements.

6. Interpolate the displacements from structural nodes onto aerodynamic nodes at the interface and deform the CFD volume mesh using RBF method.

7. Repeat (3)-(6) until the convergence of the displacement is achieved within each physical time step.

8. Go to (3) for the simulation of next time step.

\section{Results and Discussions}

\subsection{Model Description}

The AGARD wing 445.6 [34] is selected to validate the presented method. The weakened wing (model 3 ) is considered here. The wing is made of wood and can be treated as a single layer orthotropic material with longitudinal elastic modulus $3.1511 \mathrm{GPa}$, lateral elastic modulus $0.4162 \mathrm{GPa}$, Poisson's ratio 0.31, shear modulus $0.4392 \mathrm{GPa}$, density 


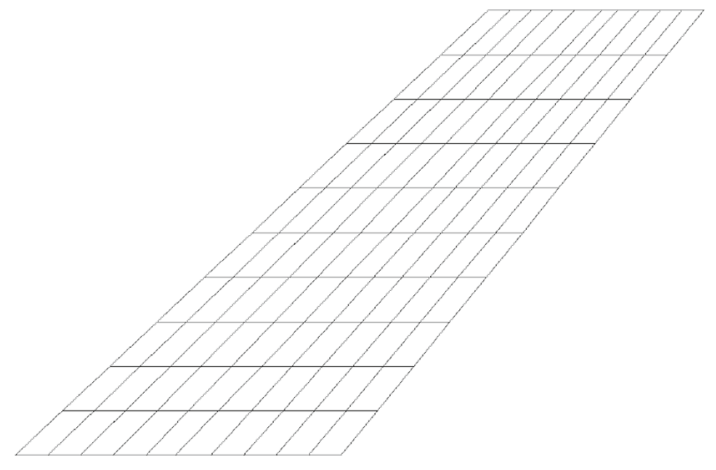

Fig. 4 Structural model using shell elements

Table 1 The first four modal frequencies

\begin{tabular}{lcc}
\hline Mode & Experiment $(\mathrm{Hz})[34]$ & Calculation $(\mathrm{Hz})$ \\
\hline 1 & 9.60 & 9.39 \\
2 & 38.10 & 39.02 \\
3 & 50.70 & 48.13 \\
4 & 98.50 & 92.54 \\
\hline
\end{tabular}

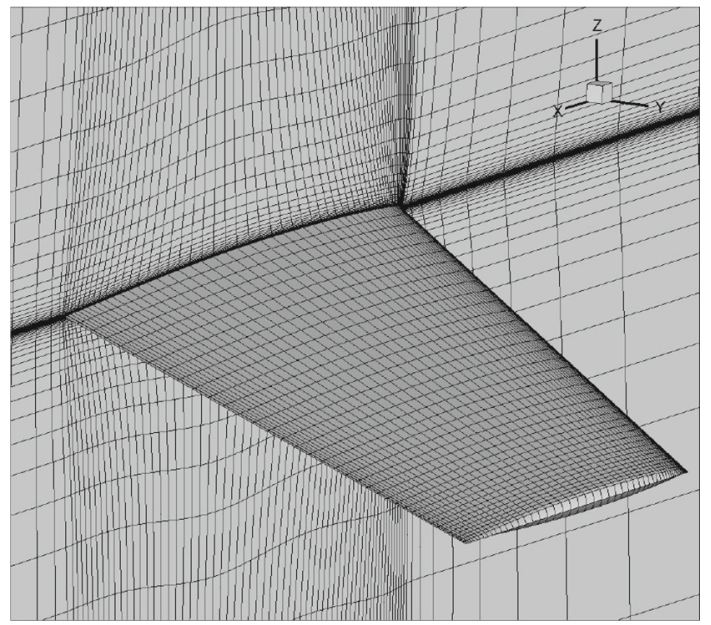

Fig. 5 CFD mesh on the wing surface and the symmetric plane

Table 2 Calculation conditions

\begin{tabular}{lll}
\hline $\mathrm{Ma}$ & $\rho\left(\mathrm{kg} / \mathrm{m}^{3}\right)$ & $\mu$ \\
\hline 0.499 & 0.4278 & 33.465 \\
0.678 & 0.2082 & 68.753 \\
0.901 & 0.09947 & 143.92 \\
0.96 & 0.06339 & 225.82 \\
1.072 & 0.05515 & 259.59 \\
\hline
\end{tabular}

$381.98 \mathrm{~kg} / \mathrm{m}^{3}$. The longitudinal direction is along the quarterchord line. The modal data in this paper are obtained by calculation using FEM, so a structural FEM model should be constructed. The structural model is built using shell elements (Fig. 4), the total number of which is 100 . Because the nodes of the shell elements are distributed on the mid-plane,

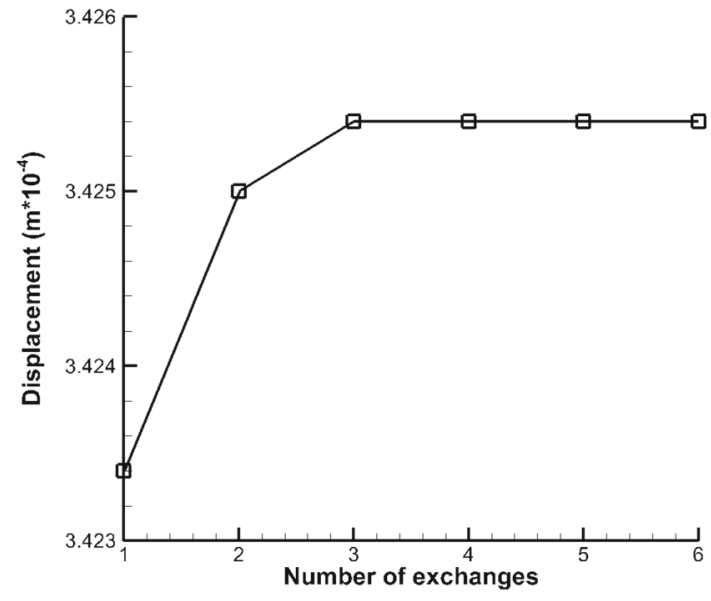

Fig. 6 Effect of the number of exchanges

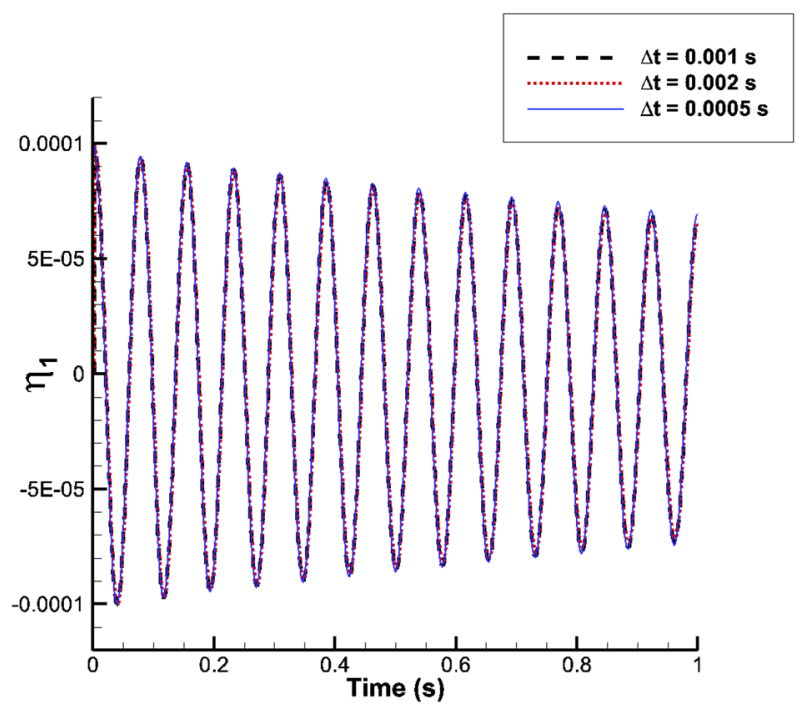

Fig. 7 Effect of time step on PIM-based coupling method, $\mathrm{Ma}=0.96$, $V=290 \mathrm{~m} / \mathrm{s}$

the RBF interpolation matrix is singular. To avoid this singularity, three-dimensional structural nodes on the wing surface are created and these nodes are connected to the mid-plane by the multiple point constraints [35]. The total number of nodes on the surface is 242. The MSC Nastran software is used to check this FEM model and calculate the dynamic characteristics. The first four modes are adopted for the aeroelastic calculations. Table 1 lists the comparison of modal frequencies, showing the calculated results are in good agreement with the measured ones [34].

Figure 5 shows the CFD mesh on the wing surface and on the symmetric plane. The structured mesh is generated with clustered boundary layer cells to resolve the viscous flow. The initial cell height is set to $4 \times 10^{-5} \mathrm{~m}$, which ensures $y^{+}$ $\approx 1$. The total number of volume cells is 0.42 million and there are 4560 elements on the surface. 


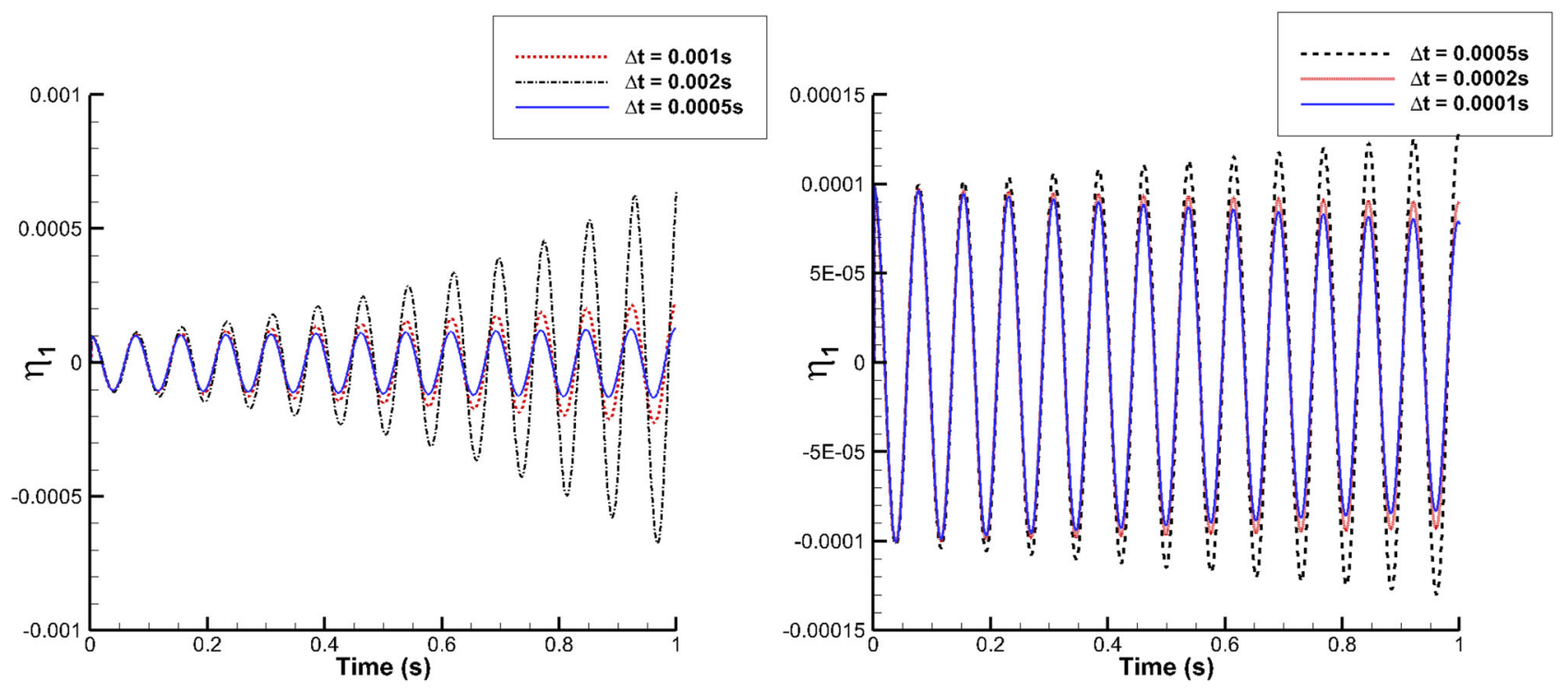

Fig. 8 Effect of time step on RK4-based coupling method, Ma $=0.96, V=290 \mathrm{~m} / \mathrm{s}$

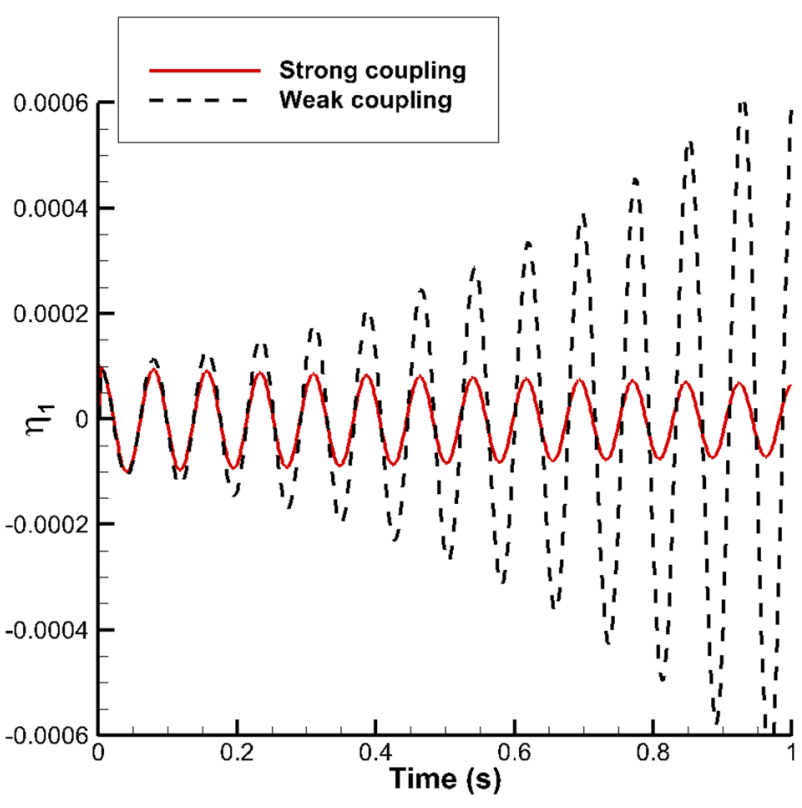

Fig. 9 Comparison between strong coupling and weak coupling using the same time step $(0.002 \mathrm{~s})$

\subsection{Flutter Calculation}

\subsubsection{Setup of Parameters}

The calculation conditions [34] are given in Table 2, where $\rho$ denotes the density of air and $\mu$ denotes the mass ratio. The flutter boundary at a specified Mach number is determined by changing the inflow velocity while keeping the density unvaried. The environment temperature is allowed to be changed so that the speed of sound and the inflow velocity can be

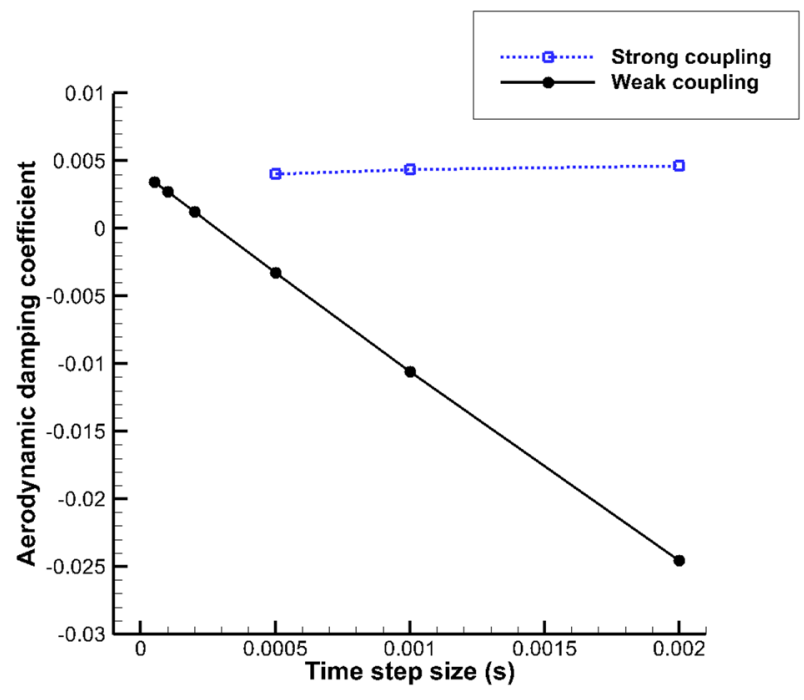

Fig. 10 Aerodynamic damping for the weak coupling and strong coupling, Ma $=0.96, V=290 \mathrm{~m} / \mathrm{s}$

changed. The environment pressure is calculated according to the equation of the state of perfect gas. The flutter simulation is started after the steady CFD calculation is converged for the rigid wing. Because the airfoil of wing 445.6 is NACA 65A004 which is symmetric [34], the initial configuration coincides with the equilibrium position. To examine the stability around the equilibrium, an initial perturbation of 0.0001 is imposed on the first mode. During the PIM-based strong coupling simulation, the data exchange is performed and the CFD mesh is updated for every 20 subiterations. Figure 6 plots the convergence history of wing displacement versus the number of exchanges within a time step, showing that it 


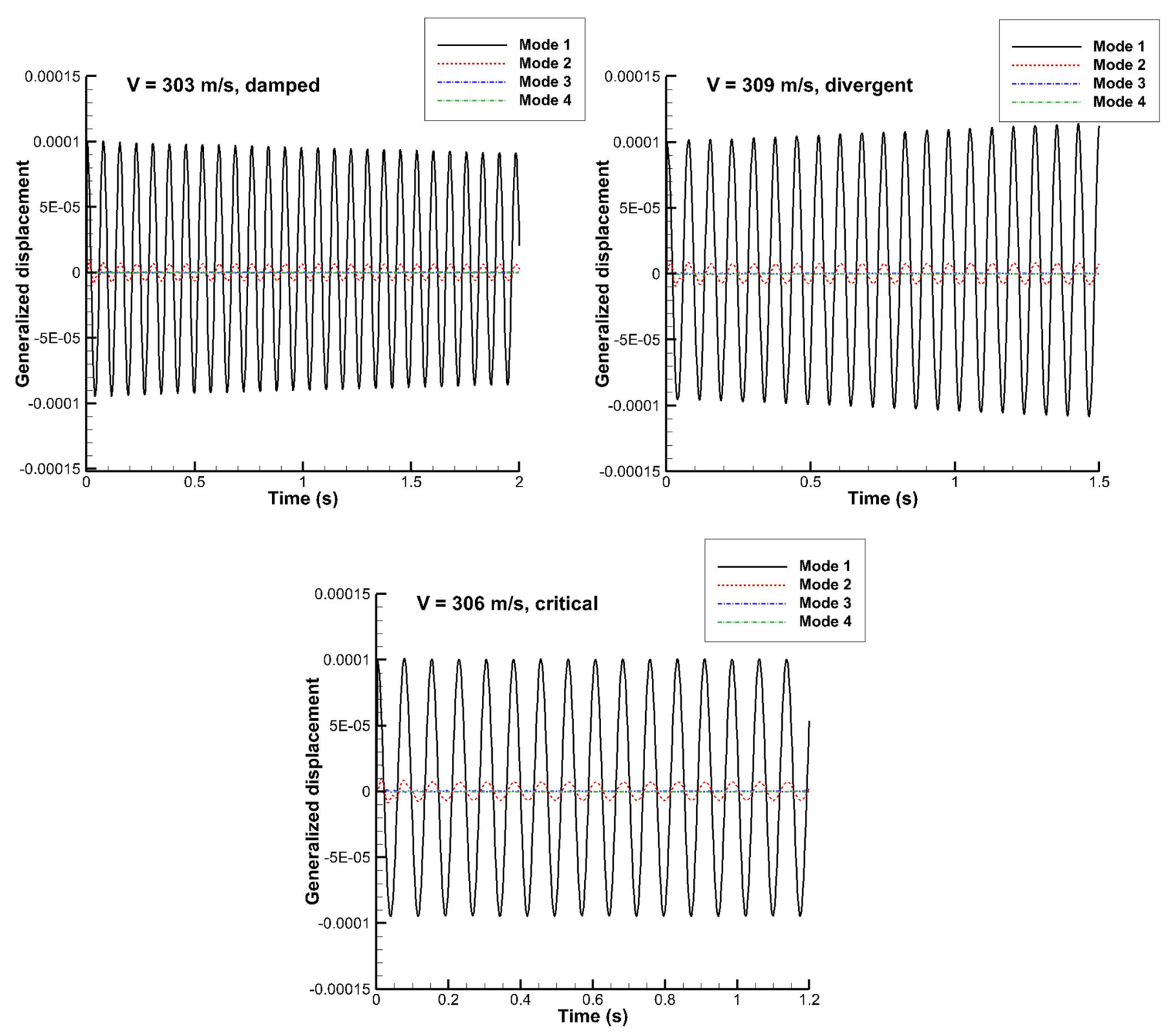

Fig. 11 Time histories of generalized displacements based on PIM, Ma $=0.96, \Delta t=0.002 \mathrm{~s}$

is converged in three steps. For the solution of flutter speed, no damping ratio is applied.

\subsubsection{Effect of Time Step}

First, the PIM-based strong coupling method is used and Fig. 7 plots the time histories of the first modal displacement $\left(\eta_{1}\right)$ using different time steps. The responses are nearly consistent with each other. And then, the traditional weak coupling approach based on the fourth-order Runge-Kutta (RK4) method is adopted to simulate the responses. This weak coupling approach has been used in flutter calculations by other studies $[14,15]$. The time histories using various time steps are shown in Fig. 8. Because the weak coupling scheme is only of first-order time accuracy, the size of time step has a great impact on the responses.

Figure 9 plots the time histories of $\eta_{1}$ using the time step of $0.002 \mathrm{~s}$ for the two methods. The strong coupling based on PIM predicts a converging result, while the weak coupling based on RK4 leads to a divergent response. Therefore, the difference between them is obvious for the same time step.

To compare the time step effect between the strong and the weak coupling methods, the aerodynamic damping coefficient $[13,20]$ is employed. Positive aerodynamic damping coefficients mean damped responses, while negative ones mean divergent responses. Figure 10 shows the convergence of aerodynamic damping coefficient of $\eta_{1}$ as a function of time step size. It is found that the aerodynamic damping coefficient shows a linear relationship with time step for the 


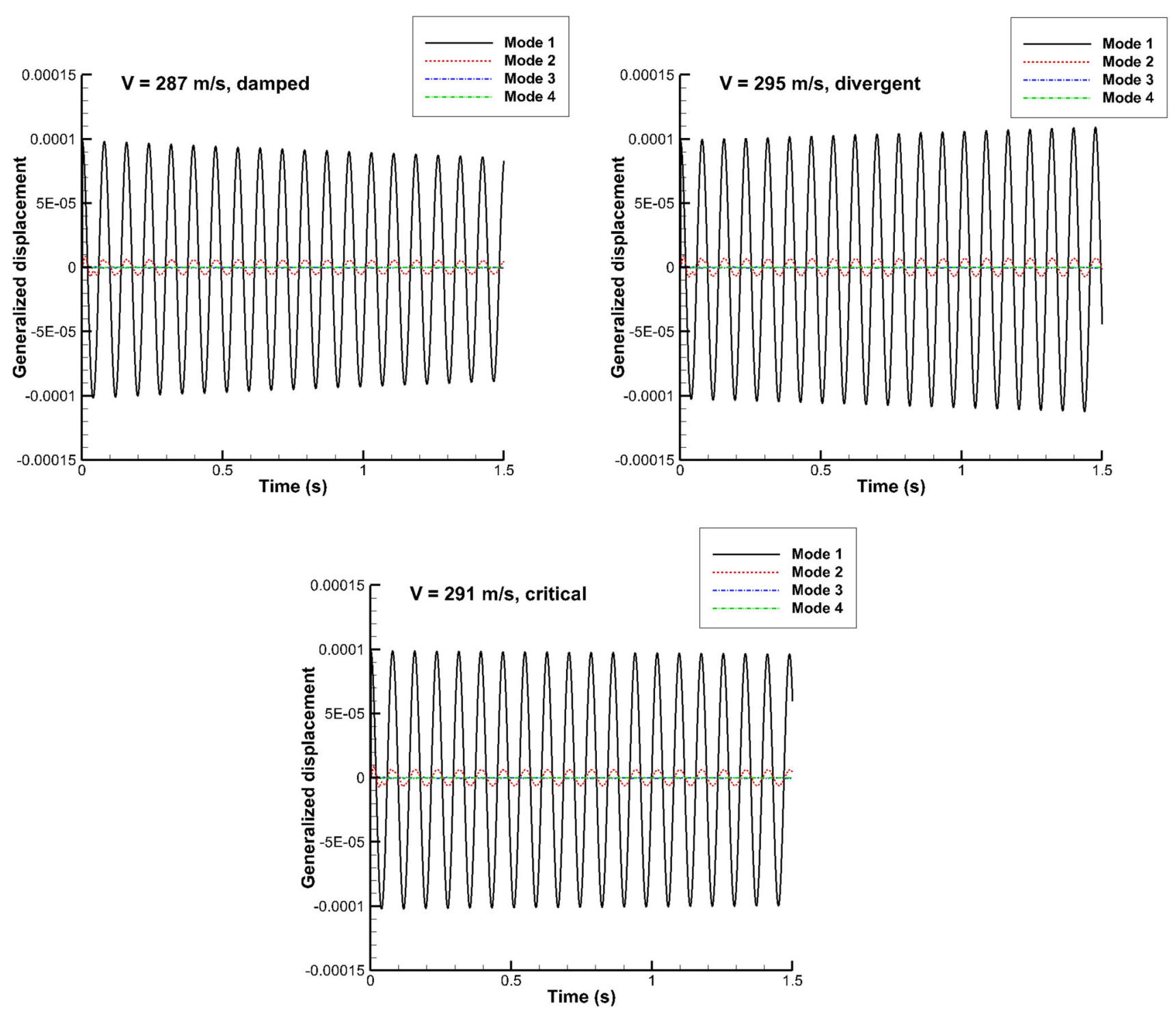

Fig. 12 Time histories of generalized displacements based on RK4, Ma $=0.96, \Delta t=0.0002 \mathrm{~s}$

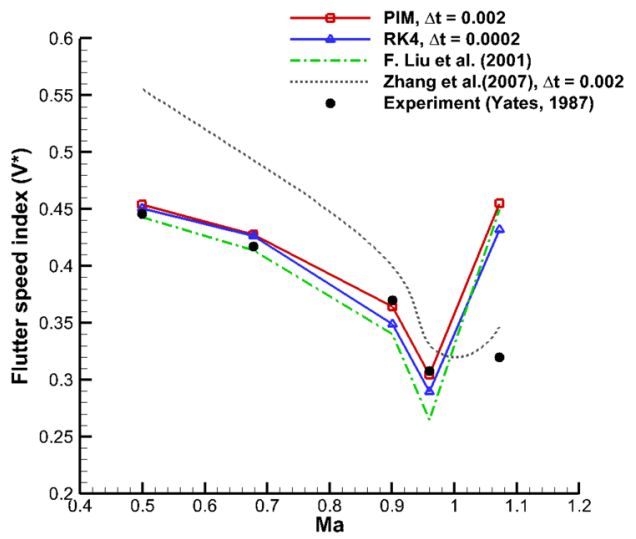

Fig. 13 Flutter speed index versus Mach number
Table 3 The comparison of computational cost

\begin{tabular}{lll}
\hline & $\begin{array}{l}\text { PIM-based strong } \\
\text { coupling }\end{array}$ & $\begin{array}{l}\text { RK4-based weak } \\
\text { coupling }\end{array}$ \\
\hline $\begin{array}{l}\text { Time step } \\
\begin{array}{l}\text { Number of time } \\
\text { steps }\end{array}\end{array}$ & $0.002 \mathrm{~s}$ & $0.0002 \mathrm{~s}$ \\
Mesh deformation & $\begin{array}{c}\text { Three times within } \\
\text { each time step }\end{array}$ & $\begin{array}{c}\text { One time within } \\
\text { each time step }\end{array}$ \\
Subiteration & $\begin{array}{c}40 \text { steps within each } \\
\text { time step }\end{array}$ & $\begin{array}{c}\text { 20 steps within each } \\
\text { time step }\end{array}$ \\
CPU time & $2.4 \mathrm{~h}$ & \begin{tabular}{c}
$10.45 \mathrm{~h}$ \\
\hline
\end{tabular}
\end{tabular}

weak coupling. The aerodynamic damping is 0.00344 and 0.00272 for the time step of $0.00005 \mathrm{~s}$ and $0.0001 \mathrm{~s}$, respectively. As the time step approaches zero, the aerodynamic 

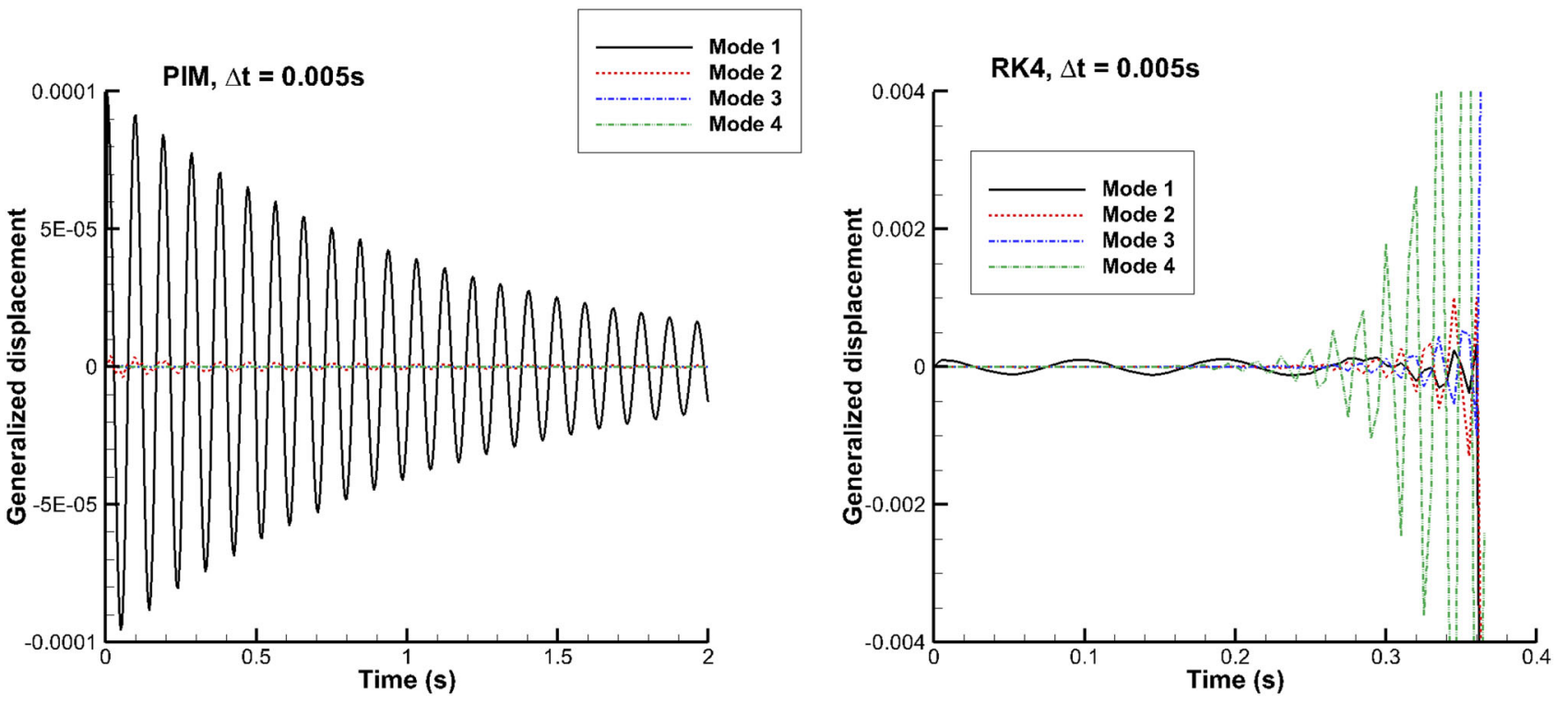

Fig. 14 The comparison of numerical stability, Ma $=0.96, V=200 \mathrm{~m} / \mathrm{s}, \Delta t=0.005 \mathrm{~s}$

damping coefficient extrapolates to 0.00404 based on the data in Fig. 10. However, the usage of a very small time step will reduce the computational efficiency significantly. In this paper, a trade-off between accuracy and efficiency is made, so the time step is set to $0.0002 \mathrm{~s}$ for the weak coupling simulations. In contrast, for the strong coupling, the aerodynamic damping coefficient changes slightly with respect to time step, indicating the presented PIM-based coupling method has better time accuracy than the traditional RK4based weak coupling.

\subsubsection{Flutter Boundary}

First, the PIM-based coupling method is used to calculate the flutter speed. The time step size is $0.002 \mathrm{~s}$, which ensures that each time period contains at least five steps for the highest mode. In Fig. 11, the time histories of generalized displacements at Mach 0.96 are plotted for three different velocities. When the velocity is $303 \mathrm{~m} / \mathrm{s}$, the response is damped. When the velocity increases to $309 \mathrm{~m} / \mathrm{s}$, the response is divergent. Only when the velocity is $306 \mathrm{~m} / \mathrm{s}$, it is a simple harmonic motion. Therefore, the flutter boundary at Mach 0.96 is $306 \mathrm{~m} / \mathrm{s}$, which is $0.97 \%$ below the measured result of $309 \mathrm{~m} / \mathrm{s}$ [34]. It demonstrates that the presented method is effective and gives high precision for the flutter predictions.

Next, the RK4-based coupling approach is used to predict the flutter boundary. Because this coupling method is only of first-order time accuracy, the time step is set to $0.0002 \mathrm{~s}$ which ensures that each time period contains at least 50 steps for the highest mode. The time histories of generalized displacements at Mach 0.96 are depicted in Fig. 12, which shows that the calculated flutter speed is $291 \mathrm{~m} / \mathrm{s}$. This calculated result is $5.83 \%$ below the measured one. Although the time step used in the RK4-based coupling calculation is one order of magnitude smaller than that used in the PIM-based coupling calculation, the error for the RK4-based coupling is still larger.

Figure 13 shows the comparison of flutter speed index $V^{*}\left(V^{*}=U_{\infty} /\left(b \omega_{\alpha} \sqrt{\mu}\right)\right.$, where $b$ is the semi-chord at the root, $\omega_{\alpha}$ is the natural frequency of the second mode, and $\mu$ refers to the mass ratio) between different methods. At Mach 0.901 and Mach 0.96, the difference between RK4 and the measurement is obvious. The PIM results show good agreement with the measurement results. Although the time step used in PIM is larger than that used in RK4, the results for PIM are still more accurate, which demonstrates that the PIM-based coupling approach is time efficient and of high fidelity. Zhang et al. [17] used the improved loose coupling approach based on linear multi-step scheme and the time step size in their work was also $0.002 \mathrm{~s}$ that is the same as PIM in the present paper. Although they have proved that their approach was also of second-order time accuracy, their predicted flutter results still deviated from the experiment obviously. Liu et al. [21] used the traditional strong coupling approach that the data communications and mesh deformations were performed in every single subiteration within each time step (Fig. 1). This approach is well known to be second-order accurate but time consuming. The comparison shown in Fig. 13 demonstrates that the present PIM results are comparable in accuracy to the traditional strong coupling approach. However, when the velocity exceeds the sound speed, the flutter boundary is overpredicted significantly for all the approaches. This deviation was also noticed by other researchers $[19,36]$ who concluded that some phys- 


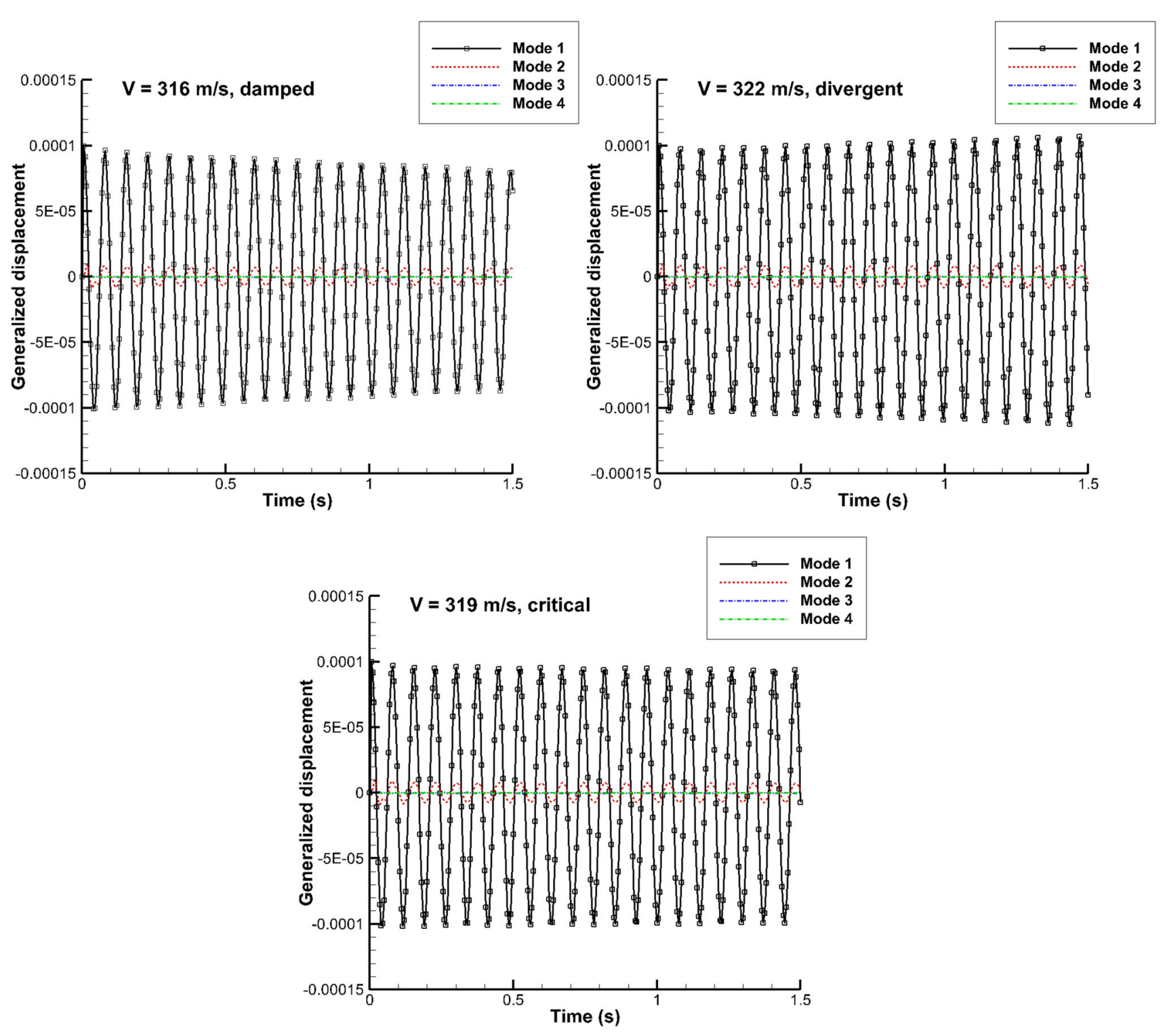

Fig. 15 Flutter calculation using PIM-based coupling with a large time step $\Delta t=0.005 \mathrm{~s}, \mathrm{Ma}=0.96$

ical conditions in the experiment might not be considered in the computations. In addition, inaccurate measurements could also lead to the deviation, because small changes in Mach number or angle of attack could generate significant changes in flutter speed in the supersonic regime.

To compare the computational efficiency between the PIM and RK4 approaches, the flutter responses are simulated for ten flutter periods and the CPU time is compared based on parallel computing using 24 processes. Table 3 lists the parameters set up for the two approaches. Because the time step for the PIM is one order of magnitude larger than the RK4, only 380 time steps are needed for ten flutter periods for PIM. Table 3 also shows that the CPU time is $2.4 \mathrm{~h}$ and $10.45 \mathrm{~h}$ for PIM and RK4, respectively. Therefore, the PIMbased strong coupling approach using a larger time step is more efficient and can reduce computational cost, although the number of subiterations and mesh deformation within each time step is greater for PIM than RK4.

\subsubsection{Numerical Stability Property}

Both PIM and RK4 are explicit schemes, but only PIM is unconditionally stable for the solution of the structural equations of motion. It means PIM allows to use an even larger time step, while RK4 does not. This difference can be illustrated with Fig. 14, in which the time step is set to $0.005 \mathrm{~s}$ corresponding to sampling the flutter period in 16 points $(N$ $=16$ ). As shown in the figure, PIM can obtain a damped motion, while the RK4 result shows that the fourth modal displacement becomes unstable first and then all modal dis- 

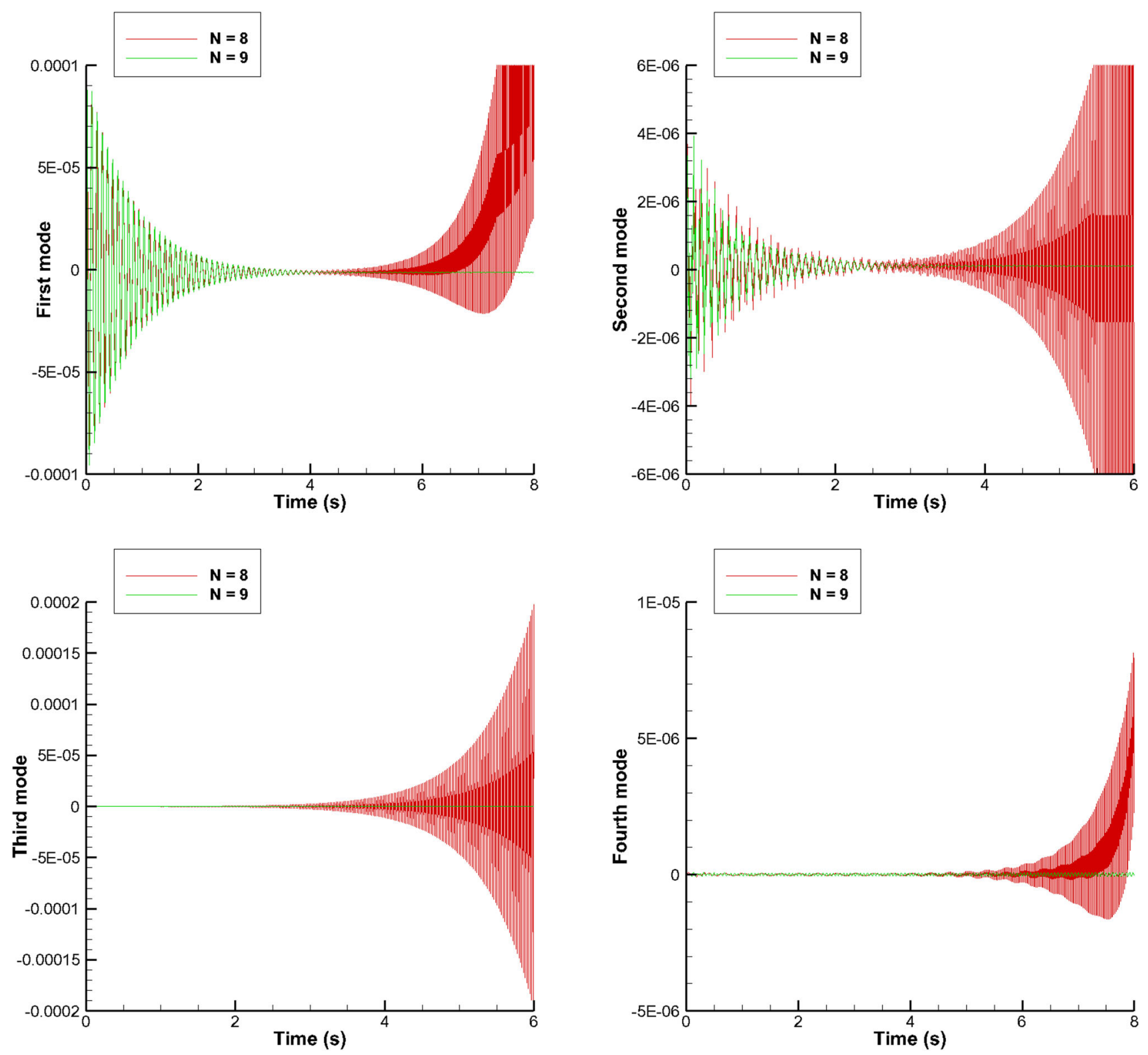

Fig. 16 Generalized displacements based on PIM computation for $N=8$ and $N=9$

placements become divergent. This time step is not small enough to consider the fourth mode, but the flutter mechanism of wing 445.6 is the coalescence of the first and the second modes. Hence, this time step should still give a reasonable flutter result. As depicted in Fig. 15, the calculated flutter speed is $319 \mathrm{~m} / \mathrm{s}$ by PIM using the time step of $0.005 \mathrm{~s}$. This result is consistent with the experiment [34]. However, further increasing the time step will reduce the flutter prediction accuracy.

Although PIM is unconditionally stable for the solution of the structural equations of motion, the dynamic aeroelastic system may still be numerically unstable if the time step is set to an unreasonably large value. Even so, PIM still shows better numerical stability property than other improved methods. For example, as shown in Fig. 16, the velocity is $280 \mathrm{~m} / \mathrm{s}$ at Mach 0.96 which is below the flutter speed, so the response should decay in time. When the time step is set to $0.0105 \mathrm{~s}$ corresponding to sampling the flutter period in eight points $(N=8)$, the response is unstable numerically. When $N$ increases to nine, the stable response can be obtained. Zhang et al. [17] also proposed two improved methods based on hybrid linear multi-step approach and improved approximate Runge-Kutta approach, which had better performance than traditional Runge-Kutta method and some other exiting methods. But their improved methods may lead to numerically unstable response for $N=26$, which is greater 


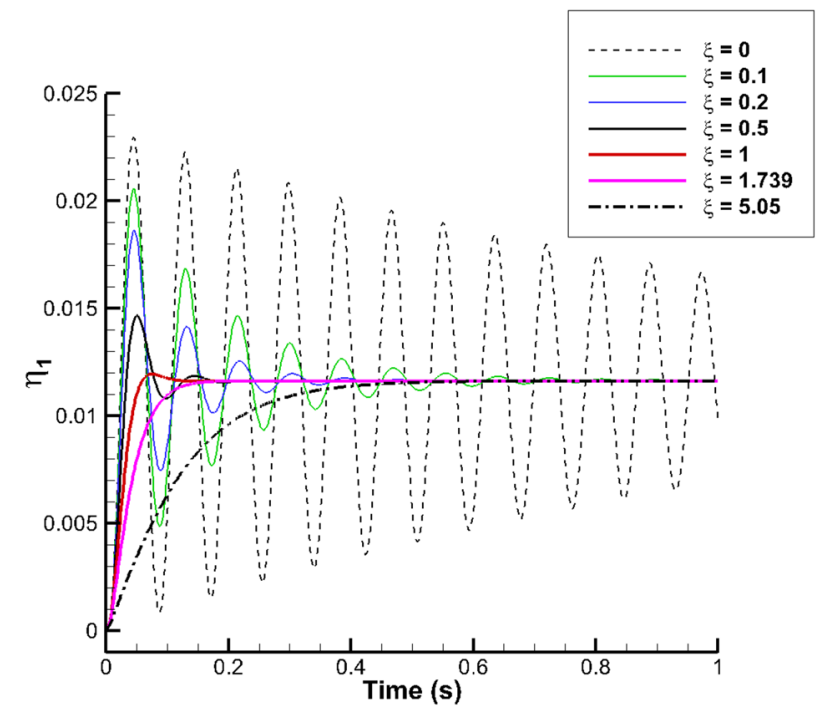

Fig. 17 The responses based on PIM using various damping ratios, $\Delta t=$ $0.002 \mathrm{~s}$

than the present work. Therefore, the presented PIM-based method has better numerical stability property than the exiting methods and allows to use a relatively large time step.

In contrast to other improved approaches based on Runge-Kutta method, central difference method, linear multi-step method and so on [12, 16, 17], the time step for PIM is not constrained by modal periods but is restricted by aerodynamic loads. To maintain the numerical stability, accuracy and efficiency, this paper recommends $16-40$ time steps per flutter period for the flutter calculation of wing 445.6.

\subsection{Static Aeroelasticity}

\subsubsection{AGARD Wing 445.6}

In this section, the static aeroelastic analyses of wing 445.6 are performed. The first four modes are used to calculate the displacements. To evaluate the effect of damping ratio on the responses, the same damping ratio is applied to all the four modes and the value is set to $0,0.1,0.2,0.5,1,1.739$ and 5.05 , respectively.

The PIM-based coupling approach is used with a time step of $0.002 \mathrm{~s}$ to simulate the responses and the time histories of the first modal displacement $\left(\eta_{1}\right)$ are shown in Fig. 17. The velocity is $250 \mathrm{~m} / \mathrm{s}$ at Mach 0.96 and the angle of attack is $2^{\circ}$. The results show that no matter how large the damping ratio is, the response is still numerically stable and converges to the same position, and that when the damping ratio is $\xi=$ 1.739 , the response approaches the steady state fast without overshoot. If the damping ratio is taken to be larger such as $\xi=5.05$, it will take more time to approach the equilibrium.

Then the RK4-based coupling approach is used to solve the problem with the same time step of $0.002 \mathrm{~s}$. The results are illustrated with Fig. 18, showing the displacements of the monitor point located in the trailing edge of the wing tip. If the damping ratio is less than or equal to one, the responses are numerically stable, which is consistent with PIM. The static displacement turns out to be $0.022 \mathrm{~m}$. If the damping ratio is increased to 1.739 , the displacement is divergent, indicating the numerical instability arises. This is because the large damping ratio will lead to the stiff problem for which a very small time step is required.

Figure 19 plots the comparisons between PIM and RK4 for two values of damping ratio. For $\xi=0.5$, both PIM and RK4
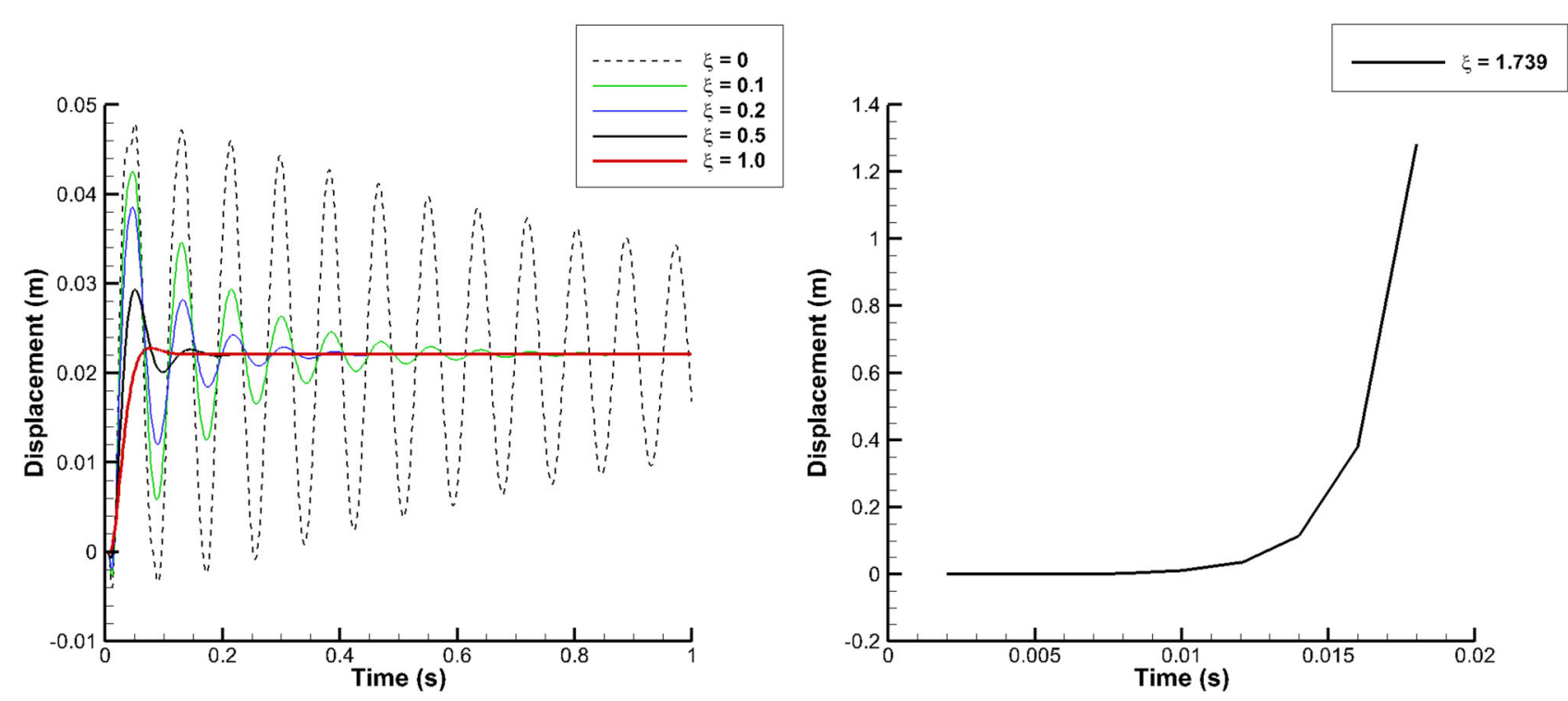

Fig. 18 The responses based on RK4 using various damping ratios, $\Delta t=0.002 \mathrm{~s}$ 

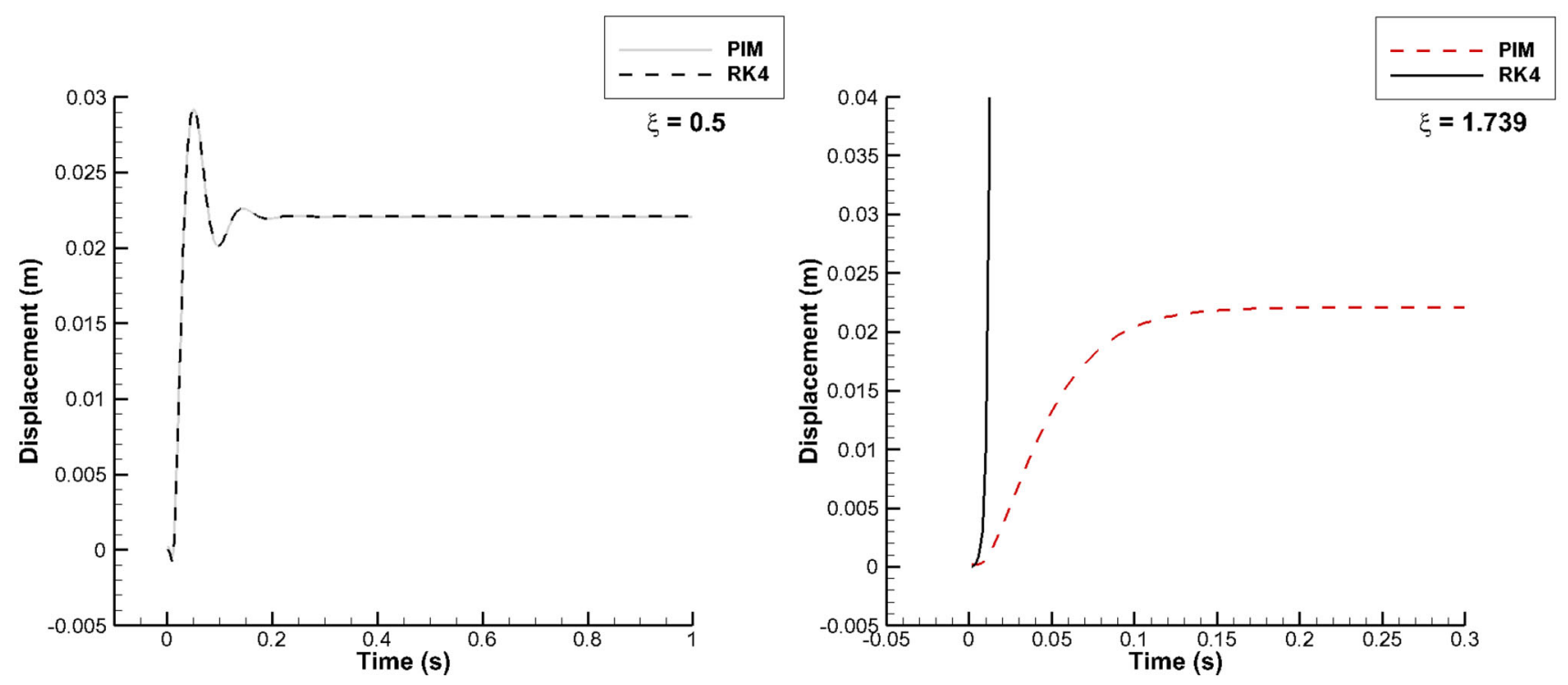

Fig. 19 Comparison between PIM and RK4, $\Delta t=0.002 \mathrm{~s}$

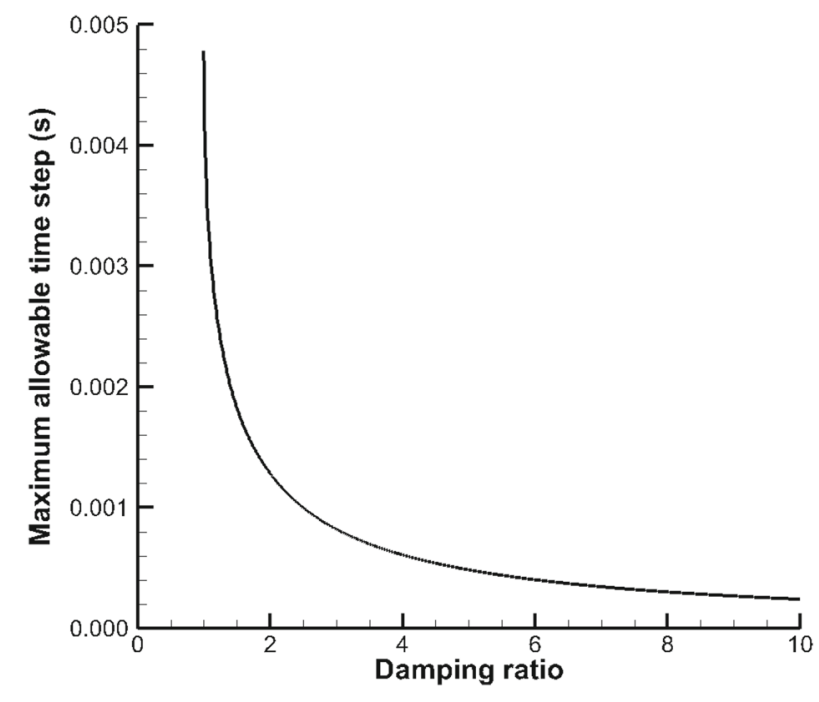

Fig. 20 Maximum allowable time step versus damping ratio for RK4 method

are numerically stable and the convergence history coincides with each other. However, for $\xi=1.739$, RK4 results in a divergent displacement, while PIM is still numerically stable.

The RK4 approach is a conditionally stable method, so the time step should be less than the maximum allowable time step which is controlled by the numerical stability condition. Since the first four modes are included, the maximum allowable time step is determined by the largest eigenvalue which is associated with the fourth mode and this time step is expressed as $2.78 /\left|\lambda_{2}\right|=2.78 /\left(\xi_{4} \omega_{4}+\omega_{4} \sqrt{\xi_{4}^{2}-1}\right)$. Figure 20 shows the relationship between the maximum allowable time step and damping ratio on the interval $(1,10)$.
The maximum allowable time step decreases rapidly as the damping ratio increases. If the damping ratio is set to 1.739 and 5.05, the corresponding stiffness ratio is 10 and 100 and the associated time step is confined to less than $0.00151 \mathrm{~s}$ and $0.000478 \mathrm{~s}$, respectively. For example, as illustrated with Fig. 21, if the damping ratio is set to 1.739 , the response is stable for the time step of $0.0015 \mathrm{~s}$ that is slightly less than $0.00151 \mathrm{~s}$. However, the fourth mode becomes unstable when the time step slightly increases to $0.0016 \mathrm{~s}$.

Because static aeroelasticity is a steady problem, the time accuracy issue does not exist and the final result is independent of the time step size. Therefore, it is suggested to choose the time step as large as possible to improve the efficiency. Even if the time step increases to $0.05 \mathrm{~s}$ which is almost half of the first modal period, PIM still predicts the stable and correct result as shown in Fig. 22. It only takes 20 steps to simulate the response for $1 \mathrm{~s}$ and the corresponding CPU time is only $8 \mathrm{~min}$ based on parallel computing using 24 processes. If RK4 with damping ratio of 1.739 is used and the time step is set to $0.0015 \mathrm{~s}$ (Fig. 21), it will take 667 steps to simulate for $1 \mathrm{~s}$ and the CPU time will increase to $105 \mathrm{~min}$, which is much greater than PIM. Therefore, PIM is more efficient than RK4 in solving static aeroelastic problems, especially when a large damping ratio is applied. This study demonstrates that the PIM-based coupling method is suitable for solving the stiff problem arising in static aeroelastic systems.

\subsubsection{Sweptback Wing}

In this section, the static aeroelastic problem of a sweptback wing [37] is solved using the developed PIM-based coupling approach. The sweptback wing is mounted on an aircraft 

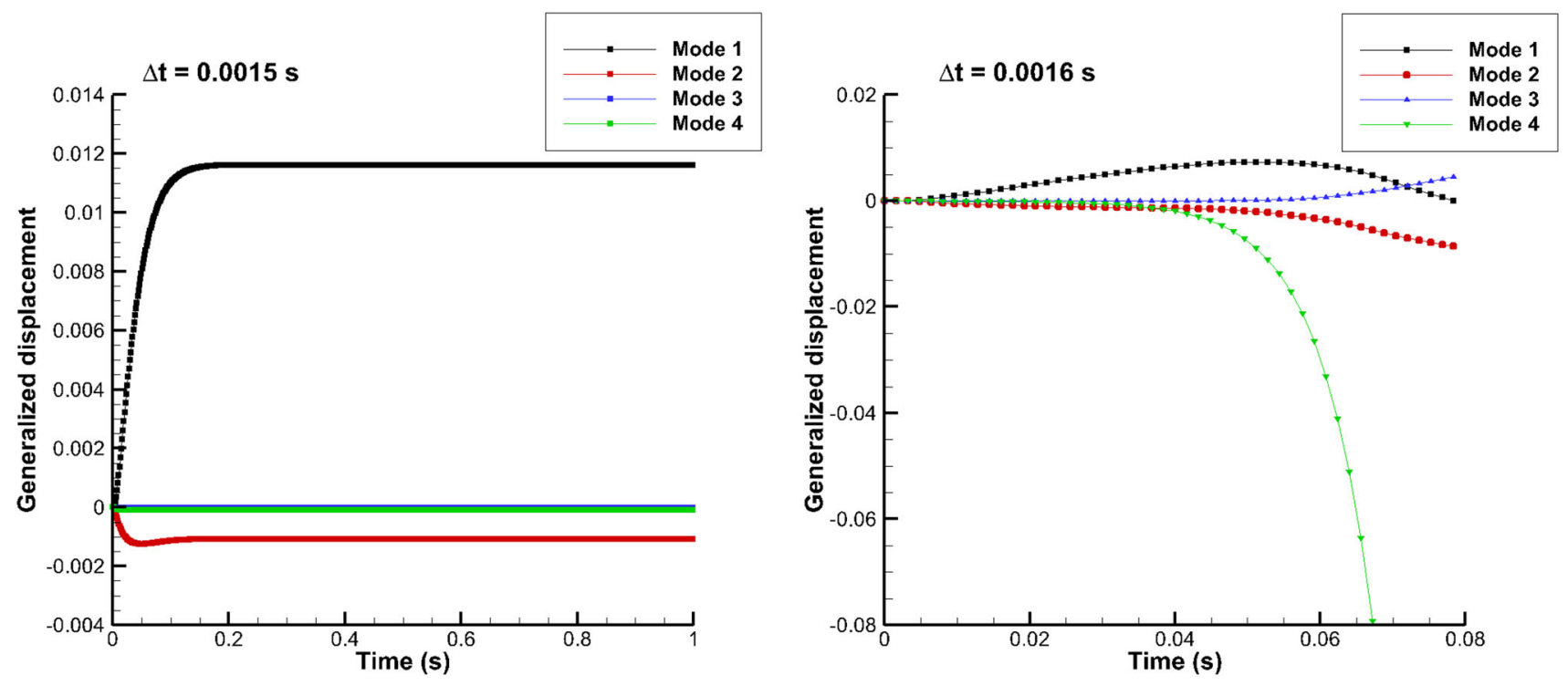

Fig. 21 The responses based on RK4 using two different time steps, $\xi=1.739$

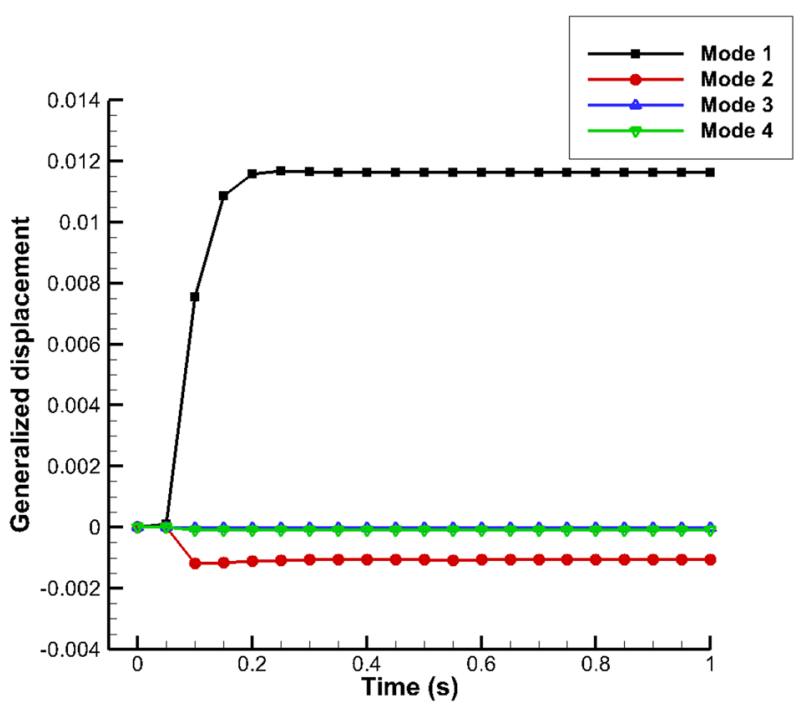

Fig. 22 The responses based on PIM for damping ratio of 1.739, $\Delta t=$ $0.05 \mathrm{~s}$

body. The wing has an aspect ratio of 9.0 and a taper ratio of 0.275 . The semi-span is $29.38 \mathrm{~m}$ and the quarter-chord sweep angle is $35^{\circ}$. Figure 23 shows the CFD mesh on the surface and on the symmetric plane. The structured mesh is generated with clustered boundary layer cells to resolve the viscous flow. The total number of volume cells is about 3.5 million.

This sweptback wing is more complex than the wing 445.6. Figure 24 shows the structural FEM model, which consists of a main spar and the skin. The main spar is built using beam elements whose stiffness decreases gradually from the wing root to the wing tip, while the skin is modeled by shell elements. The beam elements and the shell elements are con-

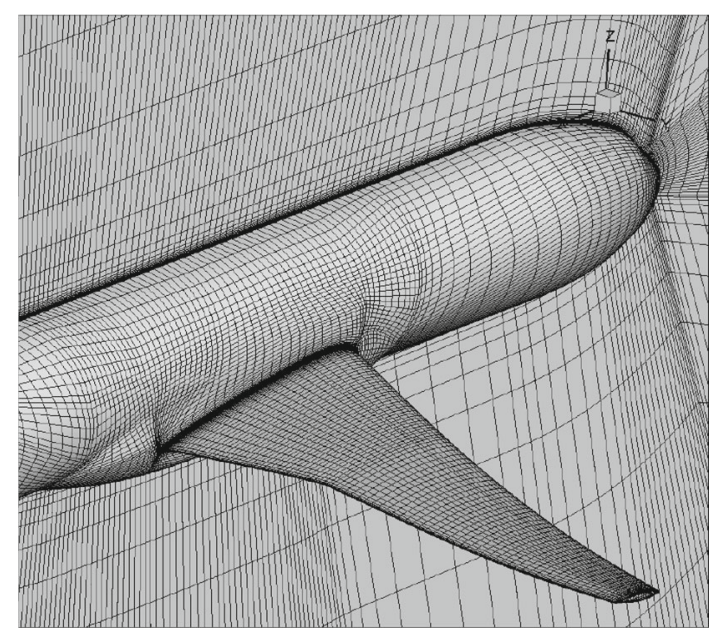

Fig. 23 CFD mesh distribution on the surface

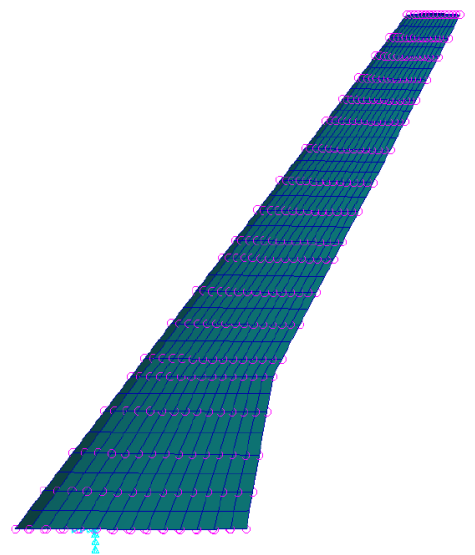

Fig. 24 The FEM model of the sweptback wing 

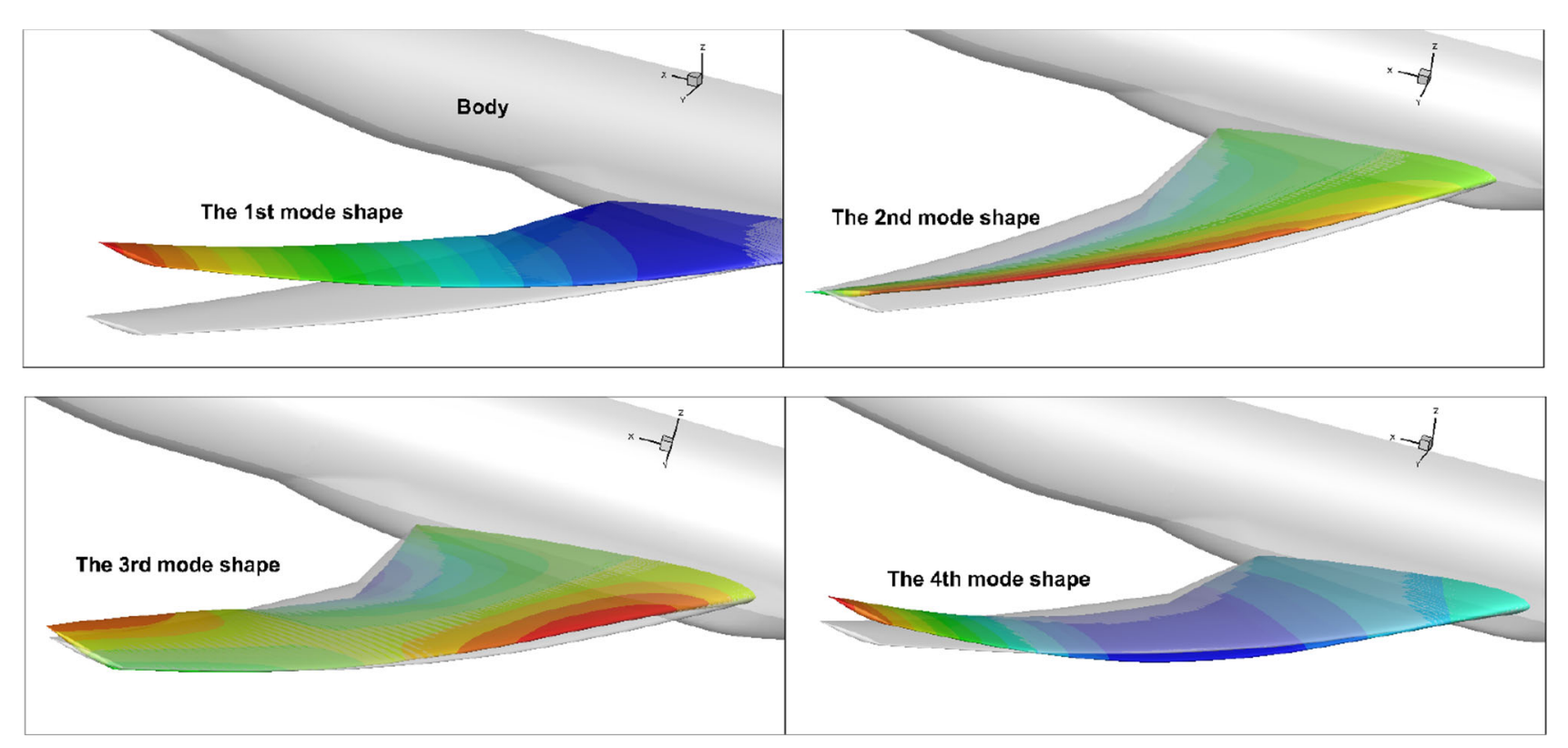

Fig. 25 The mode shapes of the sweptback wing

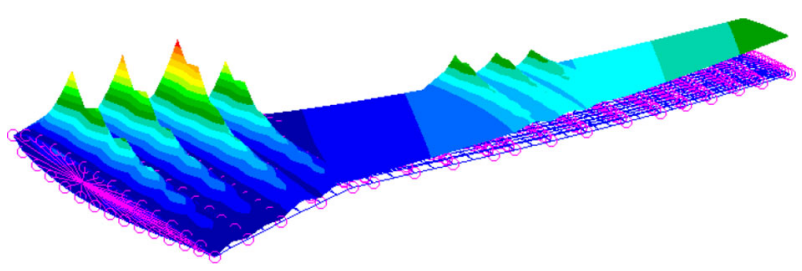

Fig. 26 Local distortion on the structural surface using flexibility method

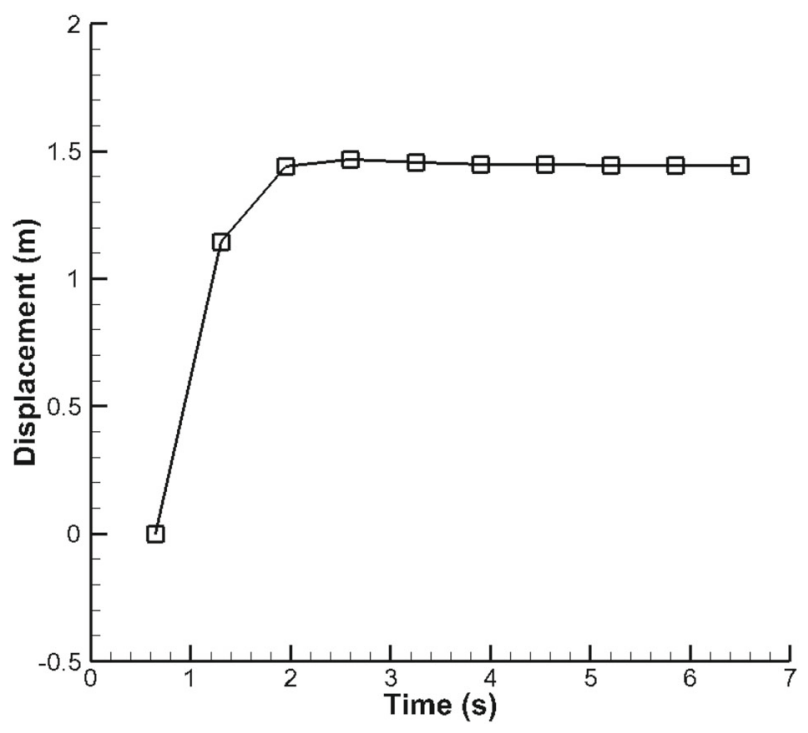

Fig. 27 Static aeroelastic simulation for the sweptback wing

nected by RBE2 elements [35]. Because the aircraft body is much more rigid than the wing, the FEM model of the body is not considered. Figure 25 shows the first four mode shapes

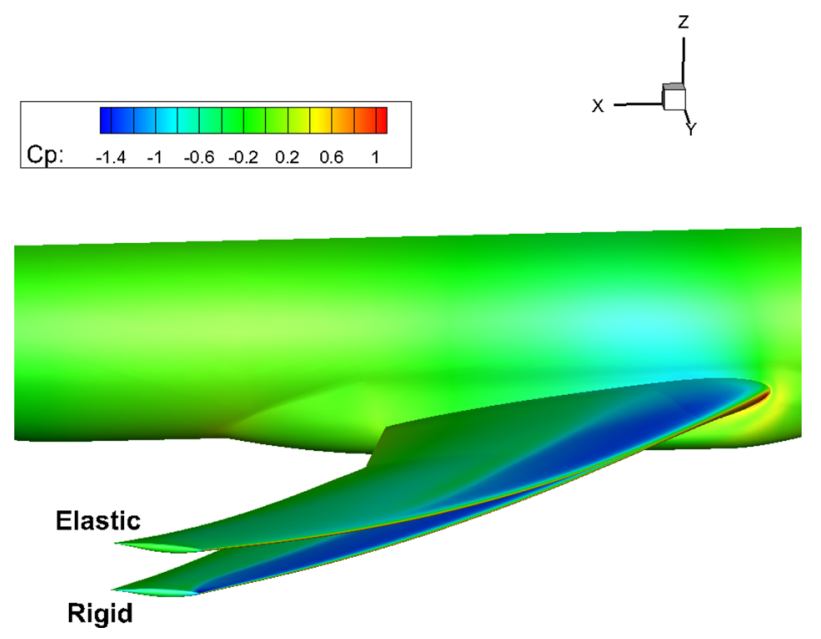

Fig. 28 Static aeroelastic deformation based on PIM

and the natural frequencies are $0.76 \mathrm{~Hz}, 1.15 \mathrm{~Hz}, 3.01 \mathrm{~Hz}$ and $3.70 \mathrm{~Hz}$, respectively. The first and the fourth modes are bending modes, while the second and the third modes are torsion modes.

The initial flow condition is provided with the free-stream Mach number of $0.8,4^{\circ}$ angle of attack and the dynamic pressure of $1905 \mathrm{~Pa}$. If the flexibility method is used, the sweptback wing produces local distortion (Fig. 26) on the skin which will lead to a failure for the static aeroelastic solution.

To resolve this problem, the PIM-based coupling approach is used. The first four modes are used to calculate the static aeroelastic displacements. The time step is set to $0.65 \mathrm{~s}$ which is about half of the first modal period and the damping ratio 
is set to 1.739 . Figure 27 shows the convergence history of a wing tip displacement. It is seen that the equilibrium is obtained within 10 steps. The static displacement turns out to be $1.445 \mathrm{~m}$. Figure 28 shows the static aeroelastic equilibrium with the pressure coefficient distribution on the surface. The deformed wing is smooth and the distortion on the skin vanishes. These results demonstrate that the developed method can effectively and efficiently overcome the distortion problem arising in the flexibility method.

\section{Conclusion}

The PIM formula is derived for the structural modal equations and the PIM-based CFD/CSD coupling method is presented for flutter predictions and static aeroelastic calculations. The AGARD wing 445.6 and a sweptback wing are considered here for the numerical study.

The time step study is performed and it is found that the aerodynamic damping coefficient varies significantly and shows a linear relationship with time step for the RK4based weak coupling, but changing slightly with time step for the PIM-based coupling. The flutter results show that the PIM-based coupling method is comparable in flutter calculation accuracy to the traditional strong coupling method and has better numerical stability property than some exiting improved methods. To maintain the numerical stability, accuracy and efficiency, this paper recommends $16-40$ time steps per flutter period for the flutter calculation of wing 445.6.

The method used to predict the flutter speed can also be used for static aeroelastic computations as long as the damping ratio is applied to the structural equations. However, large damping ratios can result in the stiff problem, for which some conventional methods need a very small time step to avoid the numerical instability. When the damping ratio is greater than one, the maximum allowable time step decreases rapidly with an increase in damping ratio for RK4. This study also shows that the PIM-based CFD/CSD coupling method is effective in solving the stiff problem arising in static aeroelastic systems and is more efficient than the RK4-based coupling approach, especially when a large damping ratio is applied. The study on the sweptback wing shows the developed PIM-based coupling method can effectively and efficiently overcome the distortion problem arising in the flexibility method.

The present work is limited to linear structures. Modern aircrafts usually have structural nonlinearities, such as geometrical nonlinearity and free-play nonlinearity. It is suggested that future work should generalize the PIM-based CFD/CSD coupling method to aeroelastic problems with structural nonlinearities.

Funding This work is supported by National Natural Science Foundation of China (No.11702298 and No.11672303).

\section{References}

1. Rodden WP, Giesing JP, Kalman TP (1972) Refinement of the nonplanar aspects of the subsonic doublet-lattice lifting surface method. J Aircr 9(1):69-73. https://doi.org/10.2514/3.44322

2. Bartels RE, Scott RC (2014) Computed and experimental flutter/LCO onset for the Boeing truss-braced wing wind tunnel model. In: Paper presented at the 44th AIAA fluid dynamics conference, AIAA 2014-2446. https://doi.org/10.2514/6.2014-2446,

3. Guimarães Neto AB, da Silva RGA, Paglione P (2014) Controlpoint-placement method for the aerodynamic correction of the vortex- and the doublet-lattice methods. Aerosp Sci Technol 37:117-129. https://doi.org/10.1016/j.ast.2014.05.007

4. Kholodar DB (2014) Nature of freeplay-induced aeroelastic oscillations. J Aircr 51(2):571-583. https://doi.org/10.2514/1.c032295

5. Bae JS, Inman DJ, Lee I (2004) Effects of structural nonlinearity on subsonic aeroelastic characteristics of an aircraft wing with control surface. J Fluids Struct 19(6):747-763. https://doi.org/10.1016/j. jfluidstructs.2004.04.005

6. Xie C, Wang L, Yang C, Liu Y (2013) Static aeroelastic analysis of very flexible wings based on non-planar vortex lattice method. Chin J Aeronaut 26(3):514-521. https://doi.org/10.1016/j.cja.2013.04. 048

7. Xie C, Yang L, Liu Y, Yang C (2018) Stability of very flexible aircraft with coupled nonlinear aeroelasticity and flight dynamics. J Aircr 55(2):862-874. https://doi.org/10.2514/1.c034162

8. Rodden WP, Taylor PF, McIntosh SC, Baker ML (1999) Further convergence studies of the enhanced doublet-lattice method. J Aircr 36(4):682-688. https://doi.org/10.2514/2.2511

9. Andrikaitis M, Fedaravičius A (2014) Modal and flutter analysis of the sailplane LAK-17B using numerical methods. Transport 29(1):84-89. https://doi.org/10.3846/16484142.2014.898217

10. Degroote J, Bathe K-J, Vierendeels J (2009) Performance of a new partitioned procedure versus a monolithic procedure in fluid-structure interaction. Comput Struct 87(11-12):793-801. https://doi. org/10.1016/j.compstruc.2008.11.013

11. Rendall TCS, Allen CB (2008) Unified fluid-structure interpolation and mesh motion using radial basis functions. Int $\mathrm{J}$ Numer Meth Eng 74(10):1519-1559. https://doi.org/10.1002/nme.2219

12. Farhat C, Lesoinne M (2000) Two efficient staggered algorithms for the serial and parallel solution of three-dimensional nonlinear transient aeroelastic problems. Comput Methods Appl Mech Eng 182(3):499-515. https://doi.org/10.1016/s0045-7825(99)00206-6

13. Jirásek A, Dalenbring M, Navrátil J (2017) Computational fluid dynamics study of benchmark supercritical wing at flutter condition. AIAA J 55(1):153-160. https://doi.org/10.2514/1.j054916

14. Liu W, Huang C, Yang G (2017) Time efficient aeroelastic simulations based on radial basis functions. J Comput Phys 330:810-827. https://doi.org/10.1016/j.jcp.2016.10.063

15. Raveh DE, Mor Yossef Y, Levy Y (2018) Analyses for the second aeroelastic prediction workshop using the EZNSS code. AIAA J 56(1):387-402. https://doi.org/10.2514/1.j055960

16. Lesoinne M, Farhat C (1998) Higher-order subiteration-free staggered algorithm for nonlinear transient aeroelastic problems. AIAA J 36(9):1754-1757. https://doi.org/10.2514/2.7555

17. Zhang W, Jiang Y, Ye Z (2007) Two better loosely coupled solution algorithms of CFD based aeroelastic simulation. Eng Appl Comp Fluid 1(4):253-262. https://doi.org/10.1080/ 19942060.2007.11015197

18. Hou G, Wang J, Layton A (2012) Numerical methods for fluid-structure interaction-a review. Commun Comput Phys 12(02):337-377. https://doi.org/10.4208/cicp.291210.290411s

19. Chen X, Zha G-C, Yang M-T (2007) Numerical simulation of 3-D wing flutter with fully coupled fluid-structural interaction. Comput 
Fluids 36(5):856-867. https://doi.org/10.1016/j.compfluid.2006. 08.005

20. Gan J-y, Im H-S, Chen X-y, Zha G-C, Pasiliao CL (2017) Delayed detached Eddy simulation of wing flutter boundary using high order schemes. J Fluids Struct 71:199-216. https://doi.org/10.1016/j. jfluidstructs.2017.03.002

21. Liu F, Cai J, Zhu Y, Tsai HM, Wong ASF (2001) Calculation of wing flutter by a coupled fluid-structure method. J Aircr 38(2):334-342. https://doi.org/10.2514/2.2766

22. van Rooij ACLM, Nitzsche J, Dwight RP (2017) Prediction of aeroelastic limit-cycle oscillations based on harmonic forcedmotion oscillations. AIAA J 55(10):3517-3529. https://doi.org/10. 2514/1.j055852

23. Alder M (2015) Development and validation of a fluid-structure solver for transonic panel flutter. AIAA J 53(12):3509-3521. https://doi.org/10.2514/1.j054013

24. Dai H, Yue X, Yuan J, Xie D, Atluri SN (2015) A comparison of classical Runge-Kutta and Henon's methods for capturing chaos and chaotic transients in an aeroelastic system with freeplay nonlinearity. Nonlinear Dyn 81(1-2):169-188. https://doi.org/10.1007/ s11071-015-1980-x

25. Lin J, Shen W, Williams FW (1995) A high precision direct integration scheme for structures subjected to transient dynamic loading. Comput Struct 56(1):113-120. https://doi.org/10.1016/ 0045-7949(94)00537-d

26. Zhong W-X (2004) On precise integration method. J Comput Appl Math 163(1):59-78. https://doi.org/10.1016/j.cam.2003.08.053

27. Gautschi W (2012) Numerical analysis, 2nd edn. Birkhäuser Boston, Cambridge

28. Chen YM, Liu JK (2014) Nonlinear aeroelastic analysis of an airfoil-store system with a freeplay by precise integration method. J Fluids Struct 46:149-164. https://doi.org/10.1016/j.jfluidstructs. 2014.01.003
29. Li Y, Hon YC (2017) Finite integration method with radial basis function for solving stiff problems. Eng Anal Boundary Elem 82:32-42. https://doi.org/10.1016/j.enganabound.2017.03.015

30. Huang C, Liu W, Yang G (2017) Numerical studies of static aeroelastic effects on grid fin aerodynamic performances. Chin $\mathbf{J}$ Aeronaut 30(4):1300-1314. https://doi.org/10.1016/j.cja.2017.04. 013

31. Jameson A (1991) Time dependent calculations using multigrid, with applications to unsteady flows past airfoils and wings. In: Paper presented at 10th Computational Fluid Dynamics Conference, AIAA 91-1596. https://doi.org/10.2514/6.1991-1596

32. Zhang W, Li X, Ye Z, Jiang Y (2015) Mechanism of frequency lock-in in vortex-induced vibrations at low Reynolds numbers. J Fluid Mech 783:72-102. https://doi.org/10.1017/jfm.2015.548

33. Rendall TCS, Allen CB (2009) Efficient mesh motion using radial basis functions with data reduction algorithms. J Comput Phys 228(17):6231-6249. https://doi.org/10.1016/j.jcp.2009.05.013

34. Yates EC (1987) AGARD standard aeroelastic configurations for dynamic response, candidate configuration I.-wing 445.6. NASATM-100492

35. MSC Nastran (2004) MSC Nastran 2004 reference manual

36. Yang G, Obayashi S, Nakamichi J (2003) Aileron buzz simulation using an implicit multiblock aeroelastic solver. J Aircr 40(3):580-589. https://doi.org/10.2514/2.3134

37. Vassberg J, Dehaan M, Rivers M, Wahls R (2008) Development of a Common Research Model for Applied CFD Validation Studies. AIAA 2008-6919. https://doi.org/10.2514/6.2008-6919

Publisher's Note Springer Nature remains neutral with regard to jurisdictional claims in published maps and institutional affiliations. 